\title{
The four-microRNA signature identified by bioinformatics analysis predicts the prognosis of nasopharyngeal carcinoma patients
}

\author{
SIWEI ZHANG ${ }^{1,2}$, WENXING YUE ${ }^{1,2}$, YAN XIE ${ }^{1,2}$, LINGZHI LIU ${ }^{1,2}$, SHEN LI $^{1,2}$, WEI DANG ${ }^{1,2}$, \\ SHUYU XIN ${ }^{1,2}$, LI YANG $^{1,2}$, XINGYU ZHAI $^{1,2}$, PENGFEI CAO ${ }^{1}$ and JIANHONG LU ${ }^{1,2}$ \\ ${ }^{1}$ NHC Key Laboratory of Carcinogenesis, Department of Hematology, Xiangya Hospital, Central South University, \\ Changsha, Hunan 410080; ${ }^{2}$ Department of Microbiology, School of Basic Medical Science, \\ Central South University, Changsha, Hunan 410078, P.R. China
}

Received March 8, 2019; Accepted July 23, 2019

DOI: $10.3892 /$ or.2019.7316

\begin{abstract}
The aim of the present study was to identify microRNAs (miRNAs) that predict the prognosis of patients with nasopharyngeal carcinoma by integrated bioinformatics analysis. First, the original microarray dataset GSE32960, including 312 nasopharyngeal carcinomas and 18 normal samples, was downloaded from the Gene Expression Omnibus database. In addition, 46 differentially expressed miRNAs (DEMs) were screened. Then, four miRNAs, including hsa-miR-142-3p, hsa-miR-150, hsa-miR-29b, and hsa-miR-29c, were obtained as prognostic markers by combining univariate Cox regression analysis with weighted gene coexpression network analysis (WGCNA). Subsequently, the risk score of 312 NPC patients from the signature of miRNAs was calculated, and patients were divided into high-risk or low-risk groups. Notably, compared with patients with low-risk scores, high-risk groups had shorter disease-free survival (DFS), overall survival (OS), and distant metastasis-free survival (DMFS). Receiver operating characteristic curve (ROC) analysis indicated that the
\end{abstract}

Correspondence to: Dr Jianhong Lu, NHC Key Laboratory of Carcinogenesis, Department of Hematology, Xiangya Hospital, Central South University, 87 Xiangya Road, Changsha, Hunan 410080, P.R. China

E-mail: jianhlu@csu.edu.cn

Abbreviations: GO, Gene Ontology; NPC, nasopharyngeal carcinoma; miRNAs, microRNAs; GEO, Gene Expression Omnibus; WGCNA, weighted gene co-expression network analysis; DEMs, differentially expressed miRNAs; PPI, protein-protein interaction; MF, molecular function; $\mathrm{BP}$, biological process; $\mathrm{CC}$, cellular component; BPA, biological pathway analysis; ROC, receiver operating characteristic; DMFS, distant metastasis-free survival; OS, overall survival; DFS, disease-free survival; DEGs, differentially expressed genes; TNM, tumor-node-metastasis; FDR, false discovery rate

Key words: nasopharyngeal carcinoma, microRNAs, prognosis, microarray, weighted gene co-expression network analysis, pathway risk score was a very effective prognostic factor. Moreover, the Search Tool for the Database for Annotation, Visualization, and Integrated Discovery (DAVID), Cytoscape, starBase, and Retrieval of Interacting Genes database (STRING) were used to establish the miRNA-mRNA correlation network and the protein-protein interaction (PPI) network. In addition, the shared genes superimposing 888 protein-coding genes targeted by four hub miRNAs and 1,601 upregulated differentially expressed mRNAs accounted for 127 and were used for subsequent gene functional enrichment analysis. In particular, biological pathway analysis indicated that these genes mainly participate in some vital pathways related to cancer pathogenesis, such as the focal adhesion, PI3K/Akt, p53, and mTOR signalling pathways. In summary, the identification of NPC patients with a four-miRNA signature may increase the prognostic value and provide reference information for precision medicine.

\section{Introduction}

Nasopharyngeal carcinoma (NPC), considered a rare tumour, has a unique geographical distribution and has the highest prevalence in Southeast Asia, such as southeastern China, including Guangdong and Hong Kong, and in other regions (India and Thailand) (1). Distant metastasis is a leading cause of treatment failure in patients with nasopharyngeal carcinoma, in which more than $70 \%$ of them have locoregionally advanced disease (2). Even after undergoing radical treatment, $\sim 30-40 \%$ of patients with locoregionally advanced NPC ultimately develop distant metastasis (3). The present tumour-node-metastasis (TNM) system was suggested to have some limitations in predicting which patients will develop distant metastasis because it is entirely based on anatomical information (4).

Consequently, increasing the number of biomarkers has been researched to improve the prognosis and treatment efficiency of nasopharyngeal carcinoma, such as Epstein-Barr virus DNA (EBV DNA), lactate dehydrogenase (LDH), VEGF, and distant metastasis gene signature (DMGN) (5-8). However, new biomarkers that reflect tumour heterogeneity should be 
studied to determine their clinical roles and to guide personalized therapy (9). MicroRNAs (miRNAs) have been revealed to suppress or promote many cancers, such as miRNA-195, miR-BART6-3p, microRNA-150, microRNA-29c, and microRNA-29b, which are potential biomarkers for diagnosis, prognosis, and personalized treatment (10-14). However, the molecular mechanism of miRNAs in nasopharyngeal carcinoma has not been completely established (15).

In the present study, the microarray data of GSE32960 (16) and GSE12452 (17) from the Gene Expression Omnibus (GEO) dataset were applied to identify differentially expressed genes (DEGs) via integrative bioinformatics approaches. The search tools of the starBase v2.0, DAVID, STRING, and GEO databases were used to identify the core differentially expressed miRNAs (DEMs) and differentially expressed mRNAs (DEmRNAs), as well as DEM-DEmRNA interactions. In total, 46 DEMs and 2,956 DEmRNAs were identified as being aberrantly expressed. In the first analyses, four significant miRNAs were revealed to be associated with overall survival (OS), disease-free survival (DFS), and distant metastasis-free survival (DMFS) in NPC via a series of bioinformatics analyses. Notably, the risk score of four miRNAs was a greatly effective prognostic factor. Finally, a regular enrichment analysis was performed for key differentially expressed genes (DEGs) that participated in some vital pathways related to cancer pathogenesis, such as the focal adhesion, PI3K/Akt, p53, and mTOR signalling pathways. The present study aimed to identify key genes associated with the prognosis of NPC and to provide a theoretical basis for future molecular mechanisms.

\section{Materials and methods}

miRNA and mRNA expression data and pre-processing. The gene expression profile data (GSE32960 and GSE12452) were downloaded from the NCBI Gene Expression Omnibus (GEO) database. However, the detailed clinical data presented in Table SIV was obtained from the researchers. The GSE32960 dataset contained the microRNA profile of 312 paraffin-embedded NPC specimens and 18 normal nasopharyngeal tissues (16). The aim of the researchers of this study was to evaluate whether microRNAs can predict the survival and efficacy of concurrent chemotherapy in nasopharyngeal carcinoma (NPC) patients. The GSE12452 dataset contained the mRNA expression profile of 31 nasopharyngeal carcinomas and 10 normal healthy nasopharyngeal tissue specimens. The mRNA expression levels were measured for essentially all human genes and all latent Epstein-Barr virus (EBV) genes in nasopharyngeal carcinoma tissue samples and normal ones. The aim of the authors of this study was to analyse data for differential gene expression between nasopharyngeal carcinoma tissue samples and normal nasopharyngeal tissues and for correlations with levels of viral gene expression (17). Statistically significant DEMs between NPC samples and normal samples were obtained with the cut-off criteria of adjust $\mathrm{P}$-value [false discovery rate (FDR)] $<0.01$ and fold change $(\mathrm{FC})>2.5$. Likewise, with a cut-off criteria of adjust P-value $(F D R)<0.05$ and a fold change $(F C)>1.5$, statistically significant mRNAs expressing differentially were also obtained. R software (version 3.5.1, https://www.r-project. org/) and MEV (version 4.9.0, http://mev.tm4.org/) were applied to the significance analysis of differentially expressed genes.

Construction of weighted gene co-expression network. First, with the use of the systems biology method, the weighted gene co-expression network, a scale-free network from gene expression data was constructed (18). Next, a soft-thresholding power (soft-threshold, $\beta=3$ ) was selected in accordance with standard scale-free networks, with which a hierarchical clustering tree was produced using the WGCNA package (19). Then, the correlations between the 30 modules and clinical traits and P-values were assessed using $\mathrm{R}$ functions in the WGCNA package. Subsequently, the adjacency was transformed into a topological overlap matrix (TOM). In addition, an average linkage hierarchical clustering was performed on the basis of the TOM-based dissimilarity measure. Finally, a minimum size (gene group) of 10 for the gene dendrogram and a cut-line of 0.25 for the module dendrogram were selected.

Risk score. miRNAs that were associated significantly with DMFS were selected to construct a miRNA signature with the risk-score method. The risk score of each patient was the sum of the multiplication of the log-transformed normalized expression value and the regression coefficient of each gene. The risk score was used for survival analysis.

Combination of differentially expressed mRNAs with target gene prediction of DEMs. To increase the accuracy of our prediction, a Venn plot was generated to obtain the common genes of differentially expressed mRNAs (fold change $>1.5$ ) and the potential target genes of miRNAs (http://bioinformatics.psb.ugent.be/webtools/Venn/). The potential target genes of four hub DEMs (hsa-miR-142-3p, hsa-miR-150, hsa-miR-29b, and hsa-miR-29c) were analysed by using online tool starBase v2.0 (http://starbase, sysu.edu.cn/starbase2/index. php) (20).

Gene ontology (GO) analysis and pathway enrichment analysis. To further clarify the mechanism of our 127 mRNAs of interest, they were uploaded to the Database for Annotation, Visualization, and Integrated Discovery (DAVID, https://david. ncifcrf.gov/) for GO functional annotation and biological pathway analysis (21). $\mathrm{P}<0.05$ was used as the cut-off criterion. The biological significance of mRNAs was explored by GO term enrichment analysis, including molecular function (MF), biological process (BP), cellular component (CC), and biological pathway (BPA). The results of the aforementioned functional enrichment analysis were visualized via the package ('ggplot2') of R software (version 3.5.1) and GraphPad Prism (version 5.0; GraphPad Software).

PPI network and miRNA-mRNA correlation network analysis. The Search Tool for the Retrieval of Interacting Genes (STRING) database (https://string-db.org/) provides PPI information of mRNAs regarding the predicted and experimental interactions of proteins (22). Cytoscape (version 3.6.0) software was used for the construction of the PPI network under the interaction information of 101 interaction genes (23). 

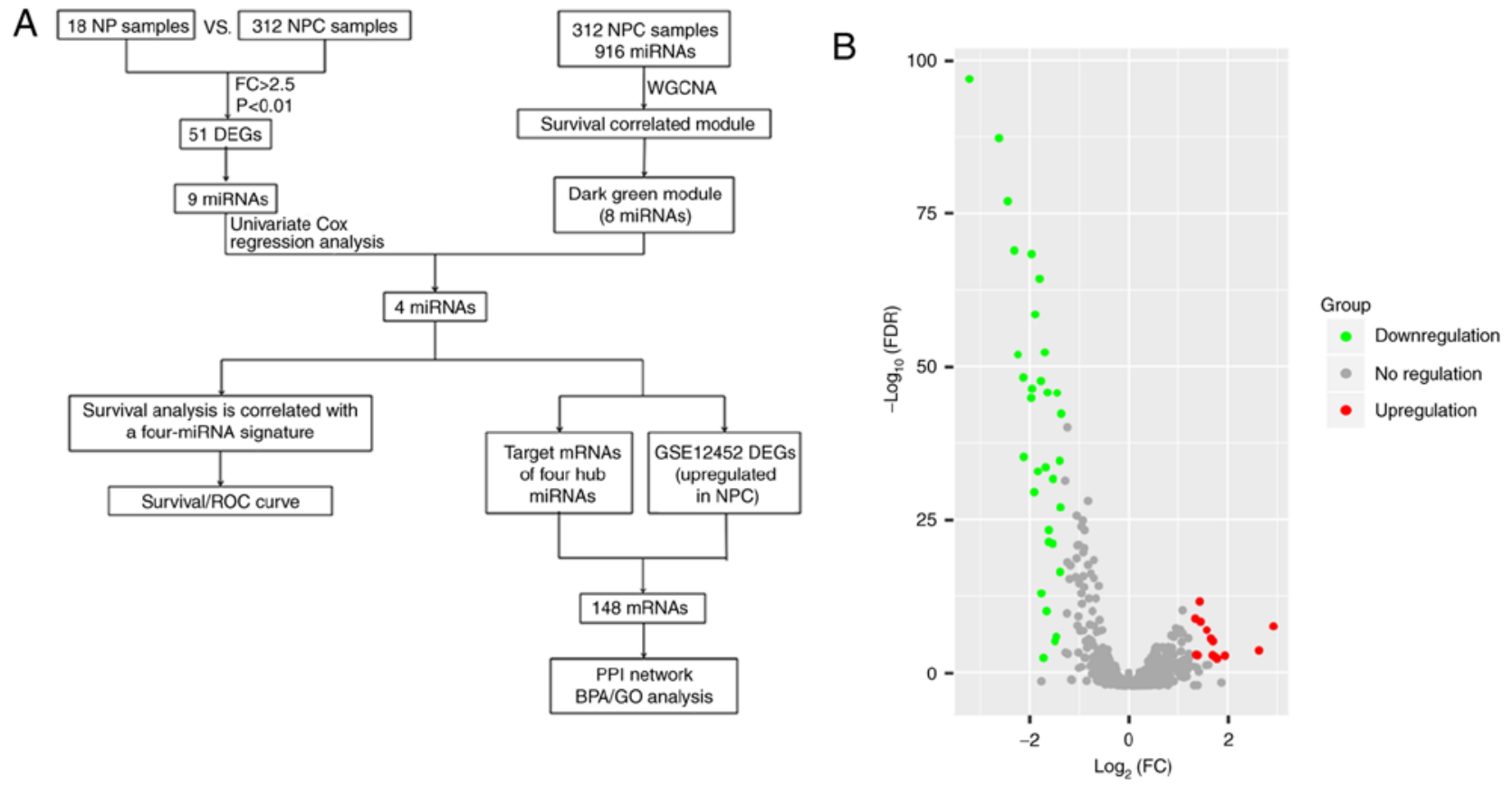

Figure 1. Filtering of differentially expressed miRNAs. (A) Data analysis pipeline to search for survival-correlated critically important miRNAs. (B) Volcano plot revealing a total of 46 miRNAs (red and green) that were differentially expressed between 312 NPC samples and 18 normal samples (cut-off criteria are fold change $>2.5$ and adjust P-value (FDR) $<0.01$ ). The red and green spots represent upregulated and downregulated miRNAs, respectively. miRNAs, microRNAs; NPC, nasopharyngeal carcinoma; DEGs, differentially expressed genes; ROC, receiver operating characteristic; WGCNA, weighted gene co-expression network analysis; PPI, protein-protein interaction; BPA; biological pathway; FDR, false discovery rate.

Superimposing four hub miRNAs with 127 mRNAs, a miRNA-mRNA correlation network was constructed utilizing Cytoscape software.

Statistical analysis. Statistical analysis was performed using SPSS 16.0 and R software 3.5.1. Graphs were generated using GraphPad Prism 5.0. Student's t-test was used to evaluate the statistical significance of the difference in the means between groups with a stringency of $\mathrm{P}<0.05$, which was considered to be significant. The $\chi^{2}$ test was used to calculate the association between two categorical variables. Kaplan-Meier survival analysis and univariate/multivariate Cox regression analysis were used to assess the expression levels of DEMs and prognostic characteristics. Receiver operating characteristic (ROC) curves were used to compare the specificity and sensitivity for the prediction of survival by the risk score, TNM stage, $\mathrm{T}$ stage, $\mathrm{N}$ stage, and sex of NPC patients. All P-values were two-sided.

\section{Results}

Identification of DEMs. The brief work-flow of this study is presented in Fig. 1A. The microarray data of GSE32960, including 312 NPC tissue samples and 18 normal tissue samples, were obtained from the NCBI-GEO database. After applying the cut-off criteria of adjust P-value (FDR) $<0.01$ and fold change $>2.5$, a total of 46 DEMs were considered statistically significant between NPC tissues and normal tissues (Table SI). The results of 32 significantly downregulated miRNAs and 14 significantly upregulated miRNAs are displayed in the volcano plot (Fig. 1B). A heat map of these 46 DEMs is presented in Fig. 2.
Construction of a weighted co-expression network and identification of key modules. First, the WGCNA package was used to cluster the miRNA expression of 312 cases of nasopharyngeal carcinoma in GSE32960 (Fig. S1). The results revealed that only the expression of GSM816316 was abnormal, which is considered an outlier sample, and thus, GSM816316 was excluded from our analysis. To ensure a scale-free network, a soft-threshold $\beta=3$ was selected to produce a hierarchical clustering tree using a WGCNA package as the soft-thresholding power and then a total of 30 modules were identified (Figs. 3 and S2). Then, a co-expression network of the associations between clinical traits and these modules was constructed using data from GSE32960, including 311 NPC samples associated with complete clinical data (Fig. 4). Notably, the dark green module was most significantly associated with survival status, such as DMFS, RFS, DFS, and OS in NPC patients. Thus, the dark green module that is most relevant to survival status was defined as a sur-module.

Identification of hub miRNAs. The 8 miRNAs belonging to the dark green module are listed in Table SII. The correlation of the 8 miRNAs of the dark green module and the survival time of patients is presented in Fig. 5A. To determine which genes were associated with distant metastasis-free survival, univariate Cox regression analysis of the DEMs individually was performed (Table SIII). Additionally, 9 miRNAs were independently significantly related to DMFS $(\mathrm{P}<0.05)$. Finally, the overlapping four miRNAs (hsa-miR-142-3p, hsa-miR-150, hsa-miR-29b, and hsa-miR-29c) were obtained as our candidate miRNAs from the two methods aforementioned (Fig. 5B). As a result, these four miRNAs were identified as potential prognostic molecules. Then, all of the tissue data was applied 

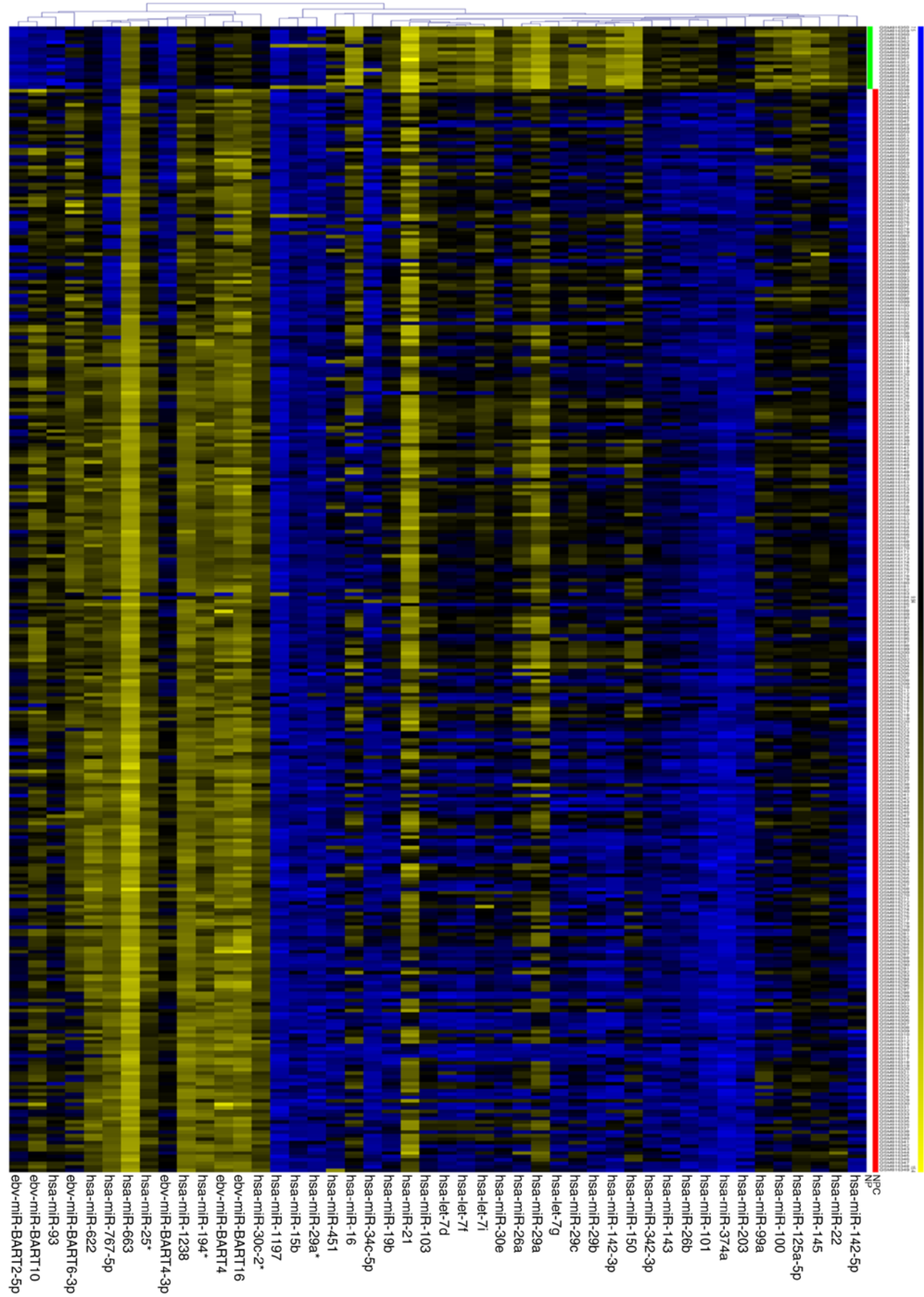

Figure 2. Hierarchical clustering analysis of the DEMs between 312 NPC and 18 normal samples. Each column represents the expression of a miRNA, and each row represents a sample: Red colour for NPC, green for normal. Yellow, upregulated miRNAs; blue, downregulated miRNAs. The * symbol in the heat map belong to the name of miRNA (an old nomenclature). miRNAs, microRNAs; NPC, nasopharyngeal carcinoma; NP, normal nasopharyngeal tissue; DEMs, differentially expressed miRNAs. 


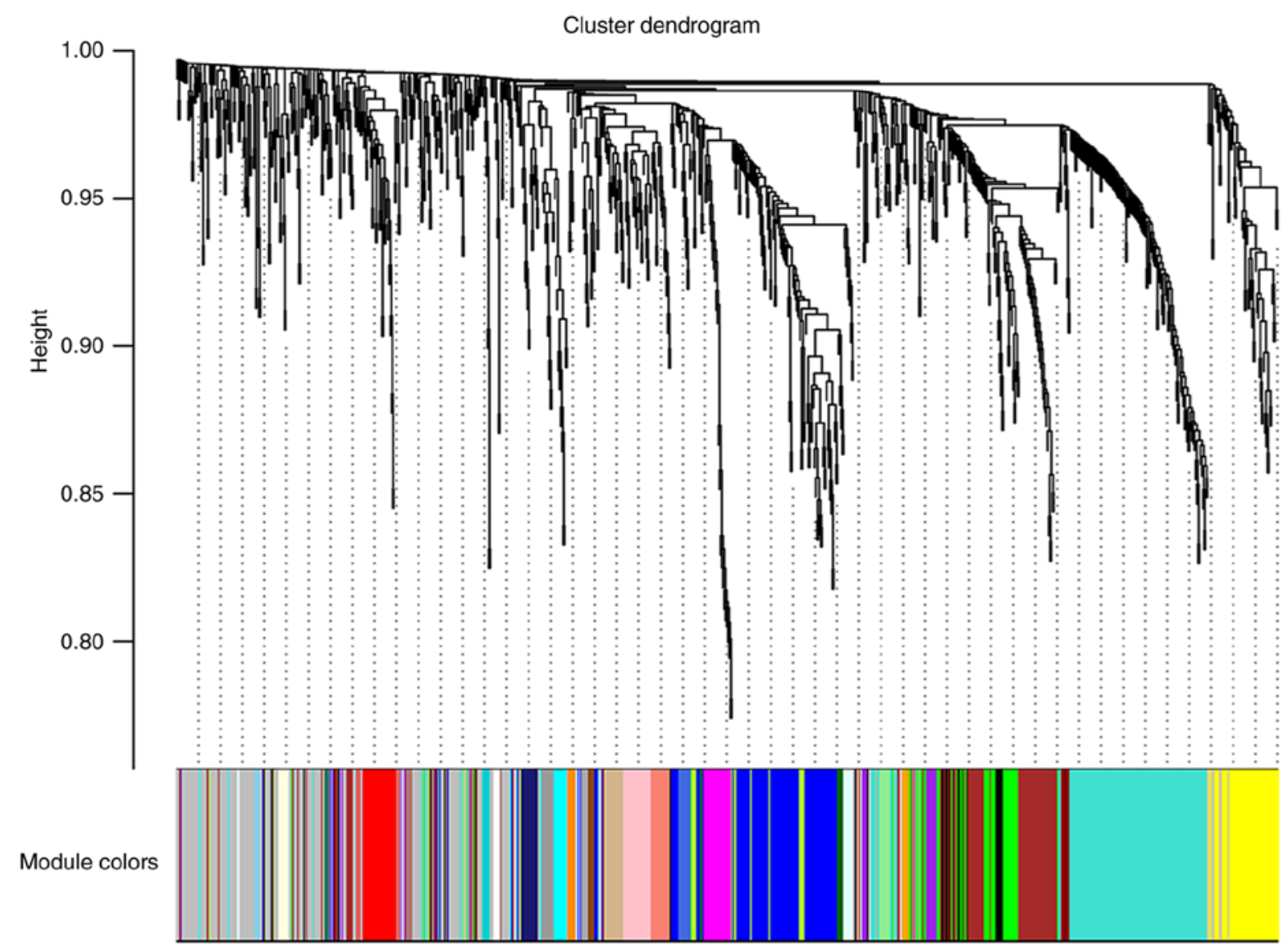

Figure 3. Clustering dendrogram and modules identified by WGCNA. miRNAs correlated with DMFS were selected by WGCNA. The clustering diagram and 30 modules for the 311 NPC tissue screening based on gene expression pattern are presented. WGCNA, weighted gene co-expression network analysis; miRNAs, microRNAs; DMFS, distant metastasis-free survival; NPC, nasopharyngeal carcinoma.

to determine the four hub miRNA expression levels between 312 NPC tissues and 18 normal tissues, and Fig. 5C revealed that compared with the normal tissues, the hub miRNAs were significantly decreased $(\mathrm{P}<0.001)$. The detailed clinical data is presented in Table SIV. Moreover, in the present study, it was determined that the four miRNAs were not only significantly downregulated but positively associated with DFS, of which miR-29c and miR-142-3p were positively associated with DMFS (Figs. S3-S5).

Feasibility analysis of miRNA as a prognostic factor for survival. We obtained a formula to calculate the risk score for every patient from the expression values of the four hub miRNAs, weighted by the regression coefficient $(24,25)$.

Risk score $=-(0.6 x$ expression value of hsa-miR-142-3p $)-$ (0.37xexpression value of hsa-miR-150)-(0.42xexpression value of hsa-miR-29b)-(0.66xexpression value of miR-29c).

With this risk score formula, the 312 NPC patients were divided into low-risk or high-risk groups with the median risk score (-19.78) as the cut-off. Furthermore, the survival difference between the two groups of patients was plotted (Fig. 6A). Notably, compared with patients with low-risk scores of the four miRNAs, patients with high-risk scores had a shorter DMFS [hazard ratio (HR) 2.276, 95\% CI, 1.403-3.692; $\mathrm{P}<0.0009]$, OS (2.119, 95\% CI, 1.337-3.357; P<0.0014), DFS
(2.183, 95\% CI, 1.457-3.271; $\mathrm{P}<0.0002)$, and RFS (1.768, 95\% CI, 0.975-3.207; $\mathrm{P}<0.0606)$. The relative clinical characteristics are presented in Table SV. To better understand which of the groupings were critical in the development of clinical outcome, univariate and multivariable Cox regression analyses were performed using a forward conditional method in view of the results of the univariate analysis. With this aforementioned analysis, it was clearly observed that the risk scores of the four miRNAs and $\mathrm{T}$ stage were independent prognostic factors for DFS, DMFS, RFS, and OS in patients with nasopharyngeal carcinoma (Tables SVI and SVII). Patients in the high-risk group had significantly higher risk scores, T-stage, and TNM stages than the low-risk group. Furthermore, to compare the sensitivity and specificity of prediction, ROC analysis was performed, and the risk score of four miRNAs exhibited a better prediction for survival than TNM stage, T stage, $\mathrm{N}$ stage and sex with regard to DMFS, OS, RFS, and DFS in patients with nasopharyngeal carcinoma (Fig. 6B). Coincidentally, the AUCs of the risk score in DMFS and DFS were both 0.69 (95\% CI, 0.63-0.75; P<0.0001). Similarly, the AUCs of the risk score in RFS and OS were both 0.68 (95\% CI, 0.62-0.74; $\mathrm{P}<0.0001)$. Thus, the risk score of the four miRNAs was an effective prognostic factor.

Combination of differentially expressed mRNAs with predicted targets of hub miRNAs. Bioinformatics analysis was applied to explore the potential correlation between miRNAs 


\begin{tabular}{|c|c|c|c|c|c|c|c|c|c|}
\hline White & $\begin{array}{l}0.014 \\
(0.8)\end{array}$ & $\begin{array}{l}-0.005 \\
(0.9)\end{array}$ & $\begin{array}{l}0.017 \\
(0.8)\end{array}$ & $\begin{array}{l}-0.017 \\
(0.8)\end{array}$ & $\begin{array}{l}0.012 \\
(0.8)\end{array}$ & $\begin{array}{c}-0.023 \\
(0.7)\end{array}$ & $\begin{array}{l}0.02 \\
(0.7)\end{array}$ & $\begin{array}{c}-0.0081 \\
(0.9)\end{array}$ & \\
\hline Magenta & $\begin{array}{l}-0.05 \\
(0.4)\end{array}$ & $\begin{array}{l}0.02 \\
(0.7)\end{array}$ & $\begin{array}{l}-0.022 \\
(0.7)\end{array}$ & $\begin{array}{l}0.016 \\
(0.8)\end{array}$ & $\begin{array}{l}-0.042 \\
(0.5)\end{array}$ & $\begin{array}{l}0.029 \\
(0.6)\end{array}$ & $\begin{array}{l}-0.026 \\
(0.6)\end{array}$ & $\begin{array}{l}0.014 \\
(0.8)\end{array}$ & 1 \\
\hline Green yellow & $\begin{array}{l}0.063 \\
(0.3)\end{array}$ & $\begin{array}{c}-0.00021 \\
\text { (1) }\end{array}$ & $\begin{array}{l}0.084 \\
(0.1)\end{array}$ & $\begin{array}{l}-0.012 \\
(0.8)\end{array}$ & $\begin{array}{l}0.065 \\
(0.3)\end{array}$ & $\begin{array}{l}-0.011 \\
(0.9)\end{array}$ & $\begin{array}{l}0.086 \\
(0.1)\end{array}$ & $\begin{array}{l}0.01 \\
(0.9)\end{array}$ & \\
\hline Blue & $\begin{array}{l}0.076 \\
(0.2)\end{array}$ & $\begin{array}{l}-0.032 \\
(0.6)\end{array}$ & $\begin{array}{l}0.11 \\
(0.05)\end{array}$ & $\begin{array}{l}-0.079 \\
(0.2)\end{array}$ & $\begin{array}{l}0.093 \\
(0.1)\end{array}$ & $\begin{array}{c}-0.056 \\
(0.3)\end{array}$ & $\begin{array}{c}0.1 \\
(0.08)\end{array}$ & $\begin{array}{c}-0.038 \\
(0.5)\end{array}$ & \\
\hline Dark green & $\begin{array}{c}0.22 \\
(1-04)\end{array}$ & $\begin{array}{l}-0.14 \\
(0.01)\end{array}$ & $\begin{array}{c}0.23 \\
(5 e-05)\end{array}$ & $\begin{array}{l}-0.17 \\
(0.003)\end{array}$ & $\begin{array}{l}0.23 \\
(5 e-05)\end{array}$ & $\begin{array}{l}-0.17 \\
(0.003)\end{array}$ & $\begin{array}{l}0.21 \\
\left(2 e^{-}-04\right)\end{array}$ & $\begin{array}{l}-0.065 \\
(0.2)\end{array}$ & \\
\hline Sky blue & $\begin{array}{l}-0.047 \\
(0.4)\end{array}$ & $\begin{array}{l}-0.043 \\
(0.5)\end{array}$ & $\begin{array}{l}-0.018 \\
(0.7)\end{array}$ & $\begin{array}{l}-0.059 \\
(0.3)\end{array}$ & $\begin{array}{l}-0.045 \\
(0.4)\end{array}$ & $\begin{array}{l}-0.021 \\
(0.7)\end{array}$ & $\begin{array}{l}-0.013 \\
(0.8)\end{array}$ & $\begin{array}{l}-0.066 \\
(0.2)\end{array}$ & \\
\hline Cyan & $\begin{array}{c}-0.014 \\
(0.8)\end{array}$ & $\begin{array}{l}0.028 \\
(0.6)\end{array}$ & $\begin{array}{l}0.005 \\
(0.9)\end{array}$ & $\begin{array}{c}0.0088 \\
(0.9)\end{array}$ & $\begin{array}{l}-0.022 \\
(0.7)\end{array}$ & $\begin{array}{l}0.029 \\
(0.6)\end{array}$ & $\begin{array}{l}0.017 \\
(0.8)\end{array}$ & $\begin{array}{c}0.0014 \\
(1)\end{array}$ & \\
\hline Grey60 & $\begin{array}{l}-0.12 \\
(0.03)\end{array}$ & $\begin{array}{l}0.0082 \\
(0.9)\end{array}$ & $\begin{array}{l}-0.067 \\
(0.2)\end{array}$ & $\begin{array}{c}-0.019 \\
(0.7)\end{array}$ & $\begin{array}{l}-0.1 \\
(0.06)\end{array}$ & $\begin{array}{l}0.026 \\
(0.6)\end{array}$ & $\begin{array}{l}-0.071 \\
(0.2)\end{array}$ & $\begin{array}{l}-0.047 \\
(0.4)\end{array}$ & \\
\hline Midnight blue & $\begin{array}{l}-0.041 \\
(0.5)\end{array}$ & $\begin{array}{l}-0.029 \\
(0.6)\end{array}$ & $\begin{array}{l}0.023 \\
(0.7)\end{array}$ & $\begin{array}{l}-0.066 \\
(0.2)\end{array}$ & $\begin{array}{l}-0.012 \\
(0.8)\end{array}$ & $\begin{array}{l}-0.027 \\
(0.6)\end{array}$ & $\begin{array}{l}0.011 \\
(0.8)\end{array}$ & $\begin{array}{l}-0.11 \\
(0.05)\end{array}$ & -0.5 \\
\hline Royal blue & $\begin{array}{l}0.059 \\
(0.3)\end{array}$ & $\begin{array}{l}-0.052 \\
(0.4)\end{array}$ & $\begin{array}{l}0.043 \\
(0.5)\end{array}$ & $\begin{array}{l}-0.019 \\
(0.7)\end{array}$ & $\begin{array}{l}0.064 \\
(0.3)\end{array}$ & $\begin{array}{l}-0.077 \\
(0.2)\end{array}$ & $\begin{array}{l}0.041 \\
(0.5)\end{array}$ & $\begin{array}{l}0.023 \\
(0.7)\end{array}$ & \\
\hline Light yellow & $\begin{array}{l}0.012 \\
(0.8)\end{array}$ & $\begin{array}{c}-0.054 \\
(0.3)\end{array}$ & $\begin{array}{l}0.018 \\
(0.7)\end{array}$ & $\begin{array}{l}-0.053 \\
(0.4)\end{array}$ & $\begin{array}{l}0.011 \\
(0.8)\end{array}$ & $\begin{array}{l}-0.028 \\
(0.6)\end{array}$ & $\begin{array}{l}0.02 \\
(0.7)\end{array}$ & $\begin{array}{l}-0.038 \\
(0.5)\end{array}$ & \\
\hline Black & $\begin{array}{l}-0.23 \\
\left(5 e^{-05}\right)\end{array}$ & $\begin{array}{l}0.011 \\
(0.8)\end{array}$ & $\begin{array}{l}-0.17 \\
(0.002)\end{array}$ & $\begin{array}{l}0.013 \\
(0.8)\end{array}$ & $\begin{array}{l}-0.21 \\
\left(2 e^{-}-04\right)\end{array}$ & $\begin{array}{l}0.053 \\
(0.4)\end{array}$ & $\begin{array}{l}-0.18 \\
(0.001)\end{array}$ & $\begin{array}{l}-0.044 \\
(0.4)\end{array}$ & \\
\hline Green & $\begin{array}{l}-0.22 \\
(6 e-05)\end{array}$ & $\begin{array}{l}-0.0012 \\
\text { (1) }\end{array}$ & $\begin{array}{l}-0.19 \\
(7 \mathrm{e}-04)\end{array}$ & $\begin{array}{c}0.0075 \\
(0.9)\end{array}$ & $\frac{-0.21}{\left(2 e^{-}-04\right)}$ & $\begin{array}{l}0.05 \\
(0.4)\end{array}$ & $\begin{array}{l}-0.21 \\
(3 e-04)\end{array}$ & $\begin{array}{l}-0.059 \\
(0.3)\end{array}$ & \\
\hline Dark red & $\begin{array}{l}-0.18 \\
(0.001)\end{array}$ & $\begin{array}{l}0.033 \\
(0.6)\end{array}$ & $\begin{array}{l}-0.12 \\
(0.04)\end{array}$ & $\begin{array}{c}-0.0076 \\
(0.9)\end{array}$ & $\begin{array}{l}-0.15 \\
(0.006)\end{array}$ & $\begin{array}{l}0.048 \\
(0.4)\end{array}$ & $\begin{array}{l}-0.13 \\
(0.02)\end{array}$ & $\begin{array}{l}-0.052 \\
(0.4)\end{array}$ & \\
\hline Turquoise & $\begin{array}{l}-0.17 \\
(0.002)\end{array}$ & $\begin{array}{l}0.015 \\
(0.8)\end{array}$ & $\begin{array}{l}-0.13 \\
(0.02)\end{array}$ & $\begin{array}{l}0.011 \\
(0.8)\end{array}$ & $\begin{array}{l}-0.15 \\
(0.008)\end{array}$ & $\begin{array}{l}0.052 \\
(0.4)\end{array}$ & $\begin{array}{l}-0.15 \\
(0.007)\end{array}$ & $\begin{array}{l}-0.04 \\
(0.5)\end{array}$ & \\
\hline Dark turquoise & $\begin{array}{l}-0.084 \\
(0.1)\end{array}$ & $\begin{array}{l}-0.036 \\
(0.5)\end{array}$ & $\begin{array}{l}-0.055 \\
(0.3)\end{array}$ & $\begin{array}{l}-0.028 \\
(0.6)\end{array}$ & $\begin{array}{l}-0.063 \\
(0.3)\end{array}$ & $\begin{array}{l}-0.017 \\
(0.8)\end{array}$ & $\begin{array}{l}-0.072 \\
(0.2)\end{array}$ & $\begin{array}{l}-0.022 \\
(0.7)\end{array}$ & \\
\hline Light green & $\begin{array}{l}-0.16 \\
(0.006)\end{array}$ & $\begin{array}{l}0.0023 \\
\text { (1) }\end{array}$ & $\begin{array}{l}-0.11 \\
(0.05)\end{array}$ & $\begin{array}{l}0.0032 \\
\text { (1) }\end{array}$ & $\begin{array}{l}-0.13 \\
(0.02)\end{array}$ & $\begin{array}{l}0.02 \\
(0.7)\end{array}$ & $\begin{array}{l}-0.13 \\
(0.02)\end{array}$ & $\begin{array}{l}-0.011 \\
(0.8)\end{array}$ & \\
\hline Red & $\begin{array}{c}-0.035 \\
(0.5)\end{array}$ & $\begin{array}{l}0.025 \\
(0.7)\end{array}$ & $\begin{array}{l}-0.075 \\
(0.2)\end{array}$ & $\begin{array}{l}0.094 \\
(0.1)\end{array}$ & $\begin{array}{l}-0.03 \\
(0.6)\end{array}$ & $\begin{array}{l}0.04 \\
(0.5)\end{array}$ & $\begin{array}{c}-0.082 \\
(0.1)\end{array}$ & $\begin{array}{l}0.054 \\
(0.3)\end{array}$ & \\
\hline Brown & $\begin{array}{c}0.24 \\
\left(2 e^{-05}\right)\end{array}$ & $\begin{array}{l}0.02 \\
(0.7)\end{array}$ & $\begin{array}{c}0.17 \\
(0.002)\end{array}$ & $\begin{array}{l}0.037 \\
(0.5)\end{array}$ & $\begin{array}{l}0.21 \\
\left(2 e^{-1}-04\right)\end{array}$ & $\begin{array}{l}-0.033 \\
(0.6)\end{array}$ & $\begin{array}{l}0.19 \\
(90-04)\end{array}$ & $\begin{array}{l}0.097 \\
(0.09)\end{array}$ & \\
\hline Orange & $\begin{array}{c}0.13 \\
(0.02)\end{array}$ & $\begin{array}{l}-0.0069 \\
(0.9)\end{array}$ & $\begin{array}{l}0.12 \\
(0.04)\end{array}$ & $\begin{array}{l}-0.043 \\
(0.5)\end{array}$ & $\begin{array}{l}0.12 \\
(0.04)\end{array}$ & $\begin{array}{l}-0.024 \\
(0.7)\end{array}$ & $\begin{array}{l}0.13 \\
(0.02)\end{array}$ & $\begin{array}{l}-0.073 \\
(0.2)\end{array}$ & \\
\hline Purple & $\begin{array}{l}0.12 \\
(0.03)\end{array}$ & $\begin{array}{l}0.047 \\
(0.4)\end{array}$ & $\begin{array}{l}0.073 \\
(0.2)\end{array}$ & $\begin{array}{l}0.025 \\
(0.7)\end{array}$ & $\begin{array}{l}0.086 \\
(0.1)\end{array}$ & $\begin{array}{l}0.026 \\
(0.6)\end{array}$ & $\begin{array}{l}0.099 \\
(0.08)\end{array}$ & $\begin{array}{l}0.013 \\
(0.8)\end{array}$ & \\
\hline Yellow & $\begin{array}{l}-0.059 \\
(0.3)\end{array}$ & $\begin{array}{l}0.022 \\
(0.7)\end{array}$ & $\begin{array}{l}-0.097 \\
(0.09)\end{array}$ & $\begin{array}{l}0.039 \\
(0.5)\end{array}$ & $\begin{array}{l}-0.067 \\
(0.2)\end{array}$ & $\begin{array}{l}0.021 \\
(0.7)\end{array}$ & $\begin{array}{l}-0.1 \\
(0.07)\end{array}$ & $\begin{array}{l}0.025 \\
(0.7)\end{array}$ & -0.5 \\
\hline Dark grey & $\begin{array}{l}0.082 \\
(0.2)\end{array}$ & $\begin{array}{l}0.03 \\
(0.6)\end{array}$ & $\begin{array}{l}0.019 \\
(0.7)\end{array}$ & $\begin{array}{l}0.036 \\
(0.5)\end{array}$ & $\begin{array}{l}0.053 \\
(0.4)\end{array}$ & $\begin{array}{l}0.0041 \\
(0.9)\end{array}$ & $\begin{array}{l}0.034 \\
(0.5)\end{array}$ & $\begin{array}{l}0.045 \\
(0.4)\end{array}$ & \\
\hline Saddle brown & $\begin{array}{c}0.13 \\
(0.02)\end{array}$ & $\begin{array}{l}0.02 \\
(0.7)\end{array}$ & $\begin{array}{l}0.031 \\
(0.6)\end{array}$ & $\begin{array}{l}0.053 \\
(0.3)\end{array}$ & $\begin{array}{l}0.087 \\
(0.1)\end{array}$ & $\begin{array}{l}0.009 \\
(0.9)\end{array}$ & $\begin{array}{l}0.058 \\
(0.3)\end{array}$ & $\begin{array}{l}0.054 \\
(0.3)\end{array}$ & \\
\hline Dark orange & $\begin{array}{l}0.05 \\
(0.4)\end{array}$ & $\begin{array}{c}-0.0094 \\
(0.9)\end{array}$ & $\begin{array}{l}0.056 \\
(0.3)\end{array}$ & $\begin{array}{l}-0.036 \\
(0.5)\end{array}$ & $\begin{array}{l}0.039 \\
(0.5)\end{array}$ & $\begin{array}{c}-0.035 \\
(0.5)\end{array}$ & $\begin{array}{l}0.069 \\
(0.2)\end{array}$ & $\begin{array}{l}-0.032 \\
(0.6)\end{array}$ & \\
\hline Light cyan & $\begin{array}{l}-0.068 \\
(0.2)\end{array}$ & $\begin{array}{l}-0.012 \\
(0.8)\end{array}$ & $\begin{array}{l}-0.11 \\
(0.06)\end{array}$ & $\begin{array}{l}0.04 \\
(0.5)\end{array}$ & $\begin{array}{l}-0.069 \\
(0.2)\end{array}$ & $\begin{array}{l}0.0012 \\
(1)\end{array}$ & $\begin{array}{l}-0.11 \\
(0.05)\end{array}$ & $\begin{array}{l}0.054 \\
(0.3)\end{array}$ & \\
\hline Tan & $\begin{array}{l}0.075 \\
(0.2)\end{array}$ & $\begin{array}{l}-0.035 \\
(0.5)\end{array}$ & $\begin{array}{l}0.033 \\
(0.6)\end{array}$ & $\begin{array}{c}-0.0027 \\
(1)\end{array}$ & $\begin{array}{l}0.06 \\
(0.3)\end{array}$ & $\begin{array}{l}-0.044 \\
(0.4)\end{array}$ & $\begin{array}{l}0.041 \\
(0.5)\end{array}$ & $\begin{array}{l}0.043 \\
(0.5)\end{array}$ & \\
\hline Pink & $\begin{array}{l}0.16 \\
(0.006)\end{array}$ & $\begin{array}{l}-0.021 \\
(0.7)\end{array}$ & $\begin{array}{l}0.09 \\
(0.1)\end{array}$ & $\begin{array}{l}0.028 \\
(0.6)\end{array}$ & $\begin{array}{l}0.12 \\
(0.03)\end{array}$ & $\begin{array}{l}-0.027 \\
(0.6)\end{array}$ & $\begin{array}{l}0.11 \\
(0.04)\end{array}$ & $\begin{array}{l}0.08 \\
(0.2)\end{array}$ & \\
\hline Salmon & $\begin{array}{l}0.12 \\
(0.03)\end{array}$ & $\begin{array}{l}0.0025 \\
\text { (1) }\end{array}$ & $\begin{array}{l}0.058 \\
(0.3)\end{array}$ & $\begin{array}{l}0.054 \\
(0.3)\end{array}$ & $\begin{array}{l}0.084 \\
(0.1)\end{array}$ & $\begin{array}{l}0.0081 \\
(0.9)\end{array}$ & $\begin{array}{l}0.087 \\
(0.1)\end{array}$ & $\begin{array}{l}0.057 \\
(0.3)\end{array}$ & -1 \\
\hline \multirow[t]{2}{*}{ Grey } & $\begin{array}{l}0.037 \\
(0.5) \\
\end{array}$ & $\begin{array}{c}-0.032 \\
(0.6) \\
\end{array}$ & $\begin{array}{l}0.046 \\
(0.4) \\
\end{array}$ & $\begin{array}{c}-0.034 \\
(0.6) \\
\end{array}$ & $\begin{array}{l}0.048 \\
(0.4) \\
\end{array}$ & $\begin{array}{c}-0.037 \\
(0.5) \\
\end{array}$ & $\begin{array}{l}0.035 \\
(0.5) \\
\end{array}$ & $\begin{array}{c}-0.0085 \\
(0.9) \\
\end{array}$ & \\
\hline & $\begin{array}{l}\stackrel{0}{E} \\
\text { ED } \\
\text { O }\end{array}$ & 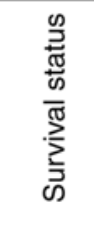 & 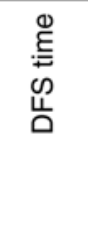 & 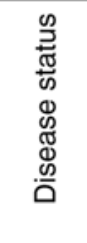 & 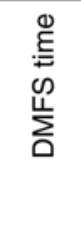 & 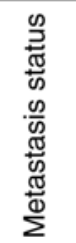 & 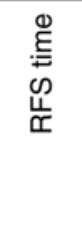 & 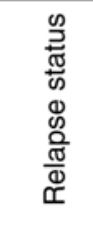 & \\
\hline
\end{tabular}

Figure 4. Module-patient trait associations. The relationship between modules and patient traits was analysed by WGCNA. Each cell contains the correlation in the first line and the P-value in the second line. The table is colour-coded by correlation according to the colour legend on the right. WGCNA, weighted gene co-expression network analysis; DFS, disease-free survival; DMFS, distant metastasis-free survival; RFS, relapse-free survival.

and mRNA expression profiles. The expression profile of GSE12452, including 31 NPC tissue samples and 10 normal tissue samples, was also obtained from the NCBI-GEO database. First, with the differential expression analysis, 2,956 mRNAs were identified as being aberrantly expressed (fold change $>1.5$, adjust $\mathrm{P}$-value (FDR) $<0.05$ ). There are two reasons for screening differentially expressed genes. One is that our main focus is on miRNAs, thus the screening of the differentially expressed miRNAs is more stringent. Another reason is that the regulation of mRNAs is complicated. Thus, the screening criteria for mRNAs was lowered in order to avoid missing genes that are not obviously altered by miRNA targeting. Of these, 1,601 mRNAs were upregulated and 1,355
mRNAs were downregulated in NPC tissues compared with normal tissues. The results of 1,355 downregulated mRNAs and 1,601 upregulated mRNAs are displayed in the volcano plot (Fig. 7A). Second, we predicted possible target genes of four hub miRNAs. The online target prediction tool starBase v2.0 was used to predict the target genes of hsa-miR-142-3p, hsa-miR-150, hsa-miR-29b, and hsa-miR-29c. The results revealed that 888 protein-coding genes were associated with the four hub miRNAs to generate 1,502 miRNA-mRNA target pairs. Finally, the 127 shared genes were obtained from 888 target genes of hub genes and 1,601 upregulated mRNAs as aforementioned (Fig. 7B; Table SVIII). As a result, 127 mRNAs were identified for the next analysis. 
A Module membership vs. gene significance Module membership vs. gene significance $C$
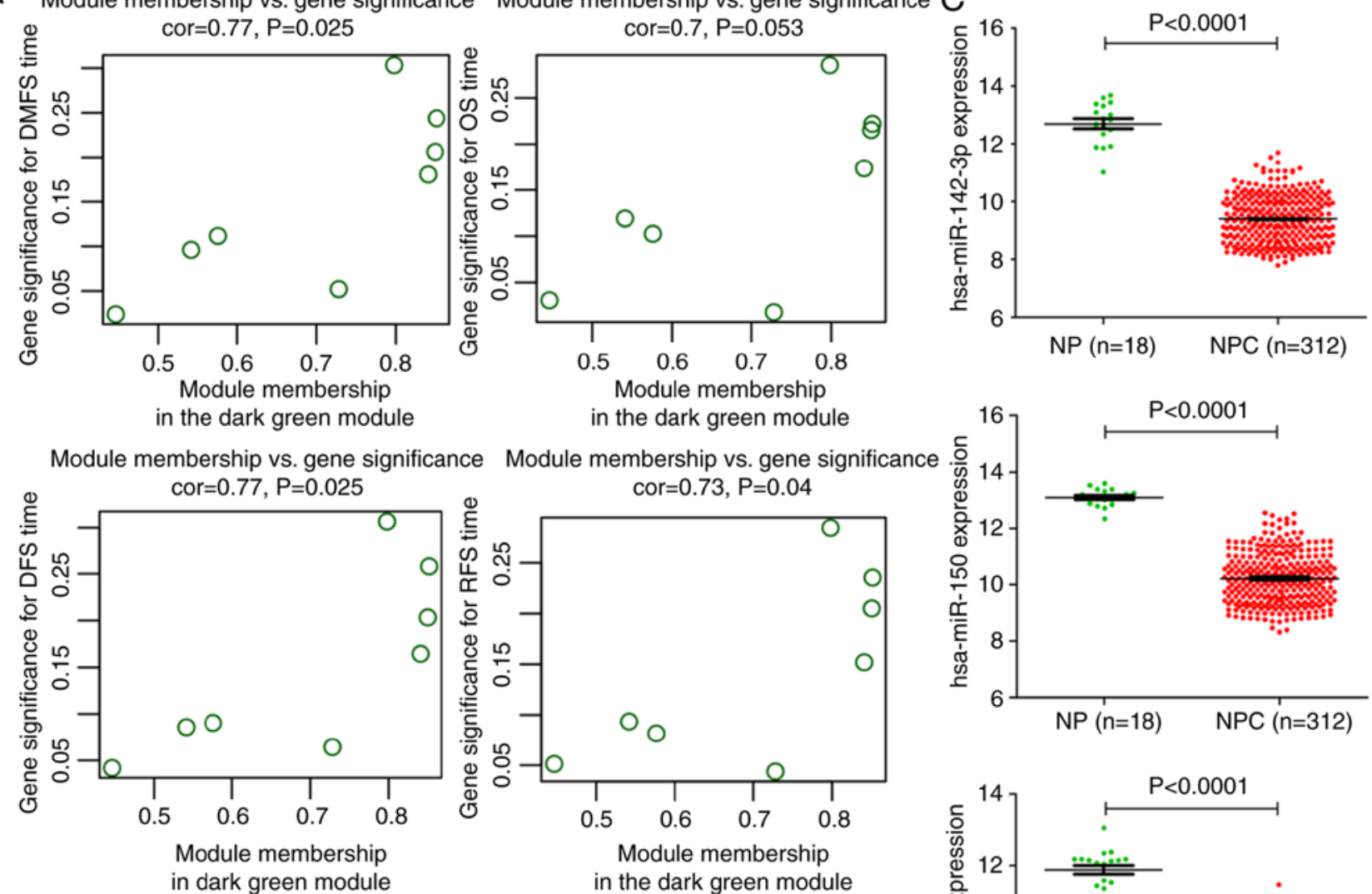

Module membership vs. gene significance

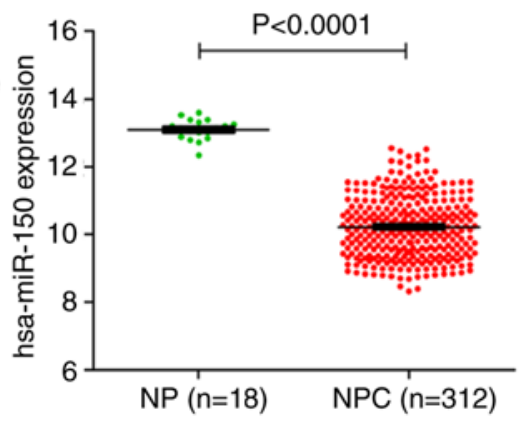

B Dark green module genes

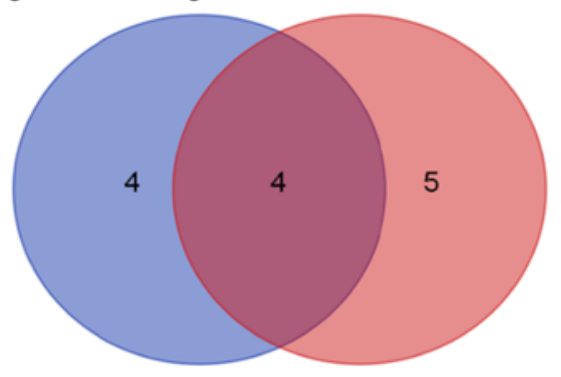

cor $=0.73, \mathrm{P}=0.04$
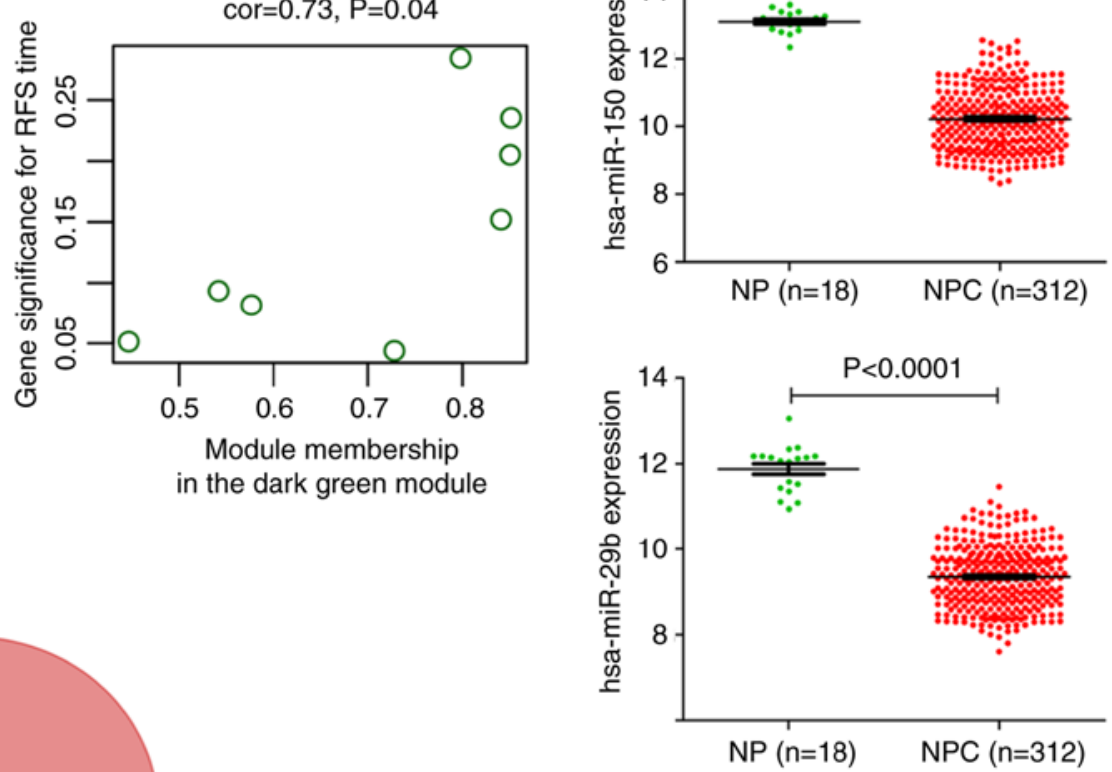

Univarite cox regression significant genes

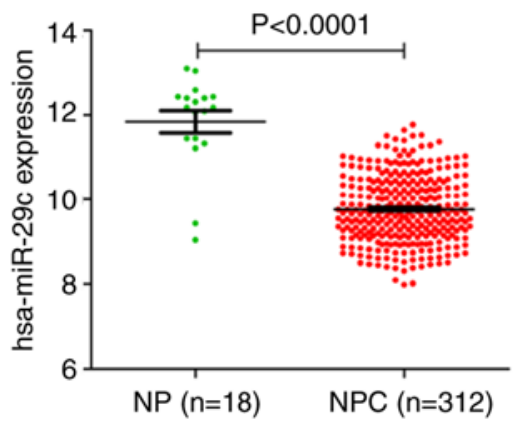

Figure 5. Relationship of the four-miRNA signature with patient survival. (A) Scatter plot for correlation between gene module membership in the dark green module (sur-module) and gene significance. The correlation analysis was performed using WGCNA package in R. (B) Venn diagram overlapping miRNAs between 'dark green module genes' and 'univariate Cox regression significant genes'. (C) Analysis of the expression levels of four hub miRNAs in 312 NPC tissues and 18 normal tissues. Data are presented as the mean \pm SEM, and the statistical significance was calculated using unpaired Student's t-tests. miRNA, microRNA; NPC, nasopharyngeal carcinoma; NP, normal nasopharyngeal tissue; DMFS, distant metastasis-free survival; OS, overall survival; DFS, disease-free survival; RFS, relapse-free survival.

The construction of the miRNA-mRNA correlation network. After merging the target genes of 4 miRNAs with 127 mRNAs, the miRNA-mRNA correlation network was constructed (Fig. 7C). In this network, circular nodes represent mRNAs, and rectangle nodes represent miRNAs. The size of the nodes is equal to the number of miRNAs corresponding to the mRNA, and the colour of the nodes represents the fold-change to mRNA.
Gene ontology $(G O)$ and pathway analysis. Functional interactions and related pathway analysis were performed on 127 mRNAs screened in the previous step to find a mechanism that affects patient survival. First, the screened 127 mRNAs were input into the String website for analysis, and the interaction information of 101 genes was obtained. Then, the previous information was imported into Cytoscape software. The number of interactions of each gene was considered as the size 

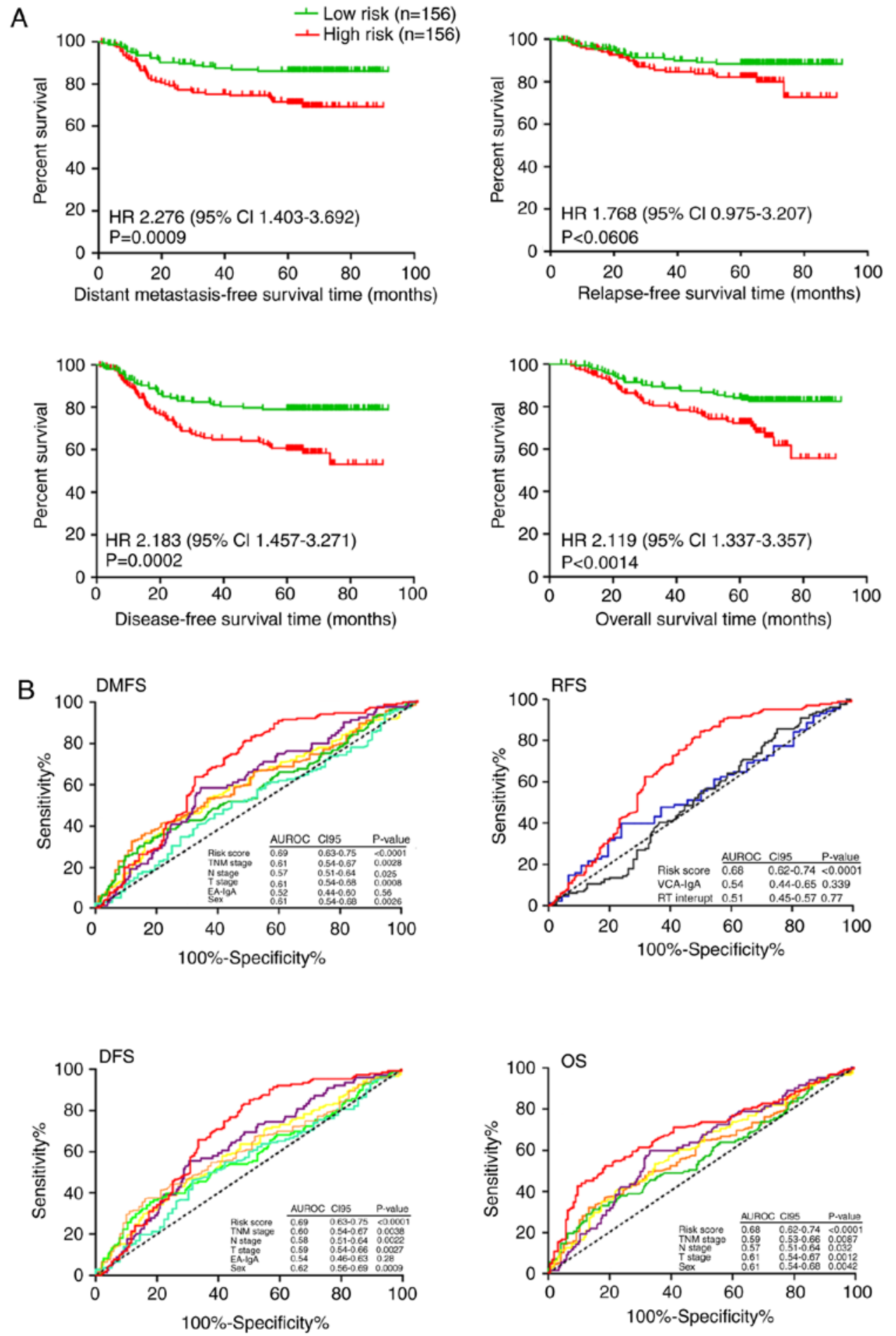

Figure 6. Feasibility analysis of miRNA as a survival prognostic factor. (A) Kaplan-Meier curves of DMFS, RFS, DFS, and OS according to the risk score of the four-miRNA signature in NPC patients. A Kaplan-Meier curve was drawn by GraphPad Prism (version 5.0). (B) Comparisons of the sensitivity and specificity for prediction of survival by the risk score of the four-microRNA signature, TNM stage, T stage, N stage, EA-IgA, VCA-IgA, sex or RT interrupt in 312 NPC patients. Survival ROC curve was drawn by GraphPad Prism (version 5.0). The HRs and P-values were calculated through an adjusted multivariate Cox regression analysis, including risk score (high risk vs. low risk), sex, age ( $\geq 45$ years vs. $<45$ years), AJCC7 N stage (stage $2-3$ vs. $0-1$ ), AJCC7 T stage (stage III-IV vs. I-II), AJCC7 TNM stage (stage III-IV vs. I-II), concurrent chemotherapy (Yes vs. No), EA-IgA ( $\geq 1: 40$ vs. 1:10-1:20 vs. $<1: 10$ ), RT boosting (Yes vs. No), RT interrupt (0 day vs. $>1$ days), sex, VCA-IgA ( $\geq 1: 640$ vs. 1:80-1:320 vs.<1:80), and WHO type (undifferentiated non-keratinizing vs. differentiated non-keratinizing vs. keratinizing squamous cell) as covariates for each analysis. miRNA, microRNA; DMFS, distant metastasis-free survival; OS, overall survival; RFS, relapse-free survival; DFS, disease-free survival; NPC, nasopharyngeal carcinoma; TNM, tumour-node-metastasis; VCA-IgA, viral capsid antigen-immunoglobulin A; EA-IgA, early antigen-immunoglobulin A; RT, radiotherapy; ROC, receiver operating characteristic; HR, hazard ratio.

of its node, and the comprehensive score of each interaction was distinguished by colour (Fig. 8). BP, CC, MF and biological pathway analyses of these 127 mRNAs are presented in
Fig. 9A and B. The GO analysis indicated that the genes were mostly enriched in the extracellular matrix organization, blood vessel development, platelet-derived growth factor binding, 
A

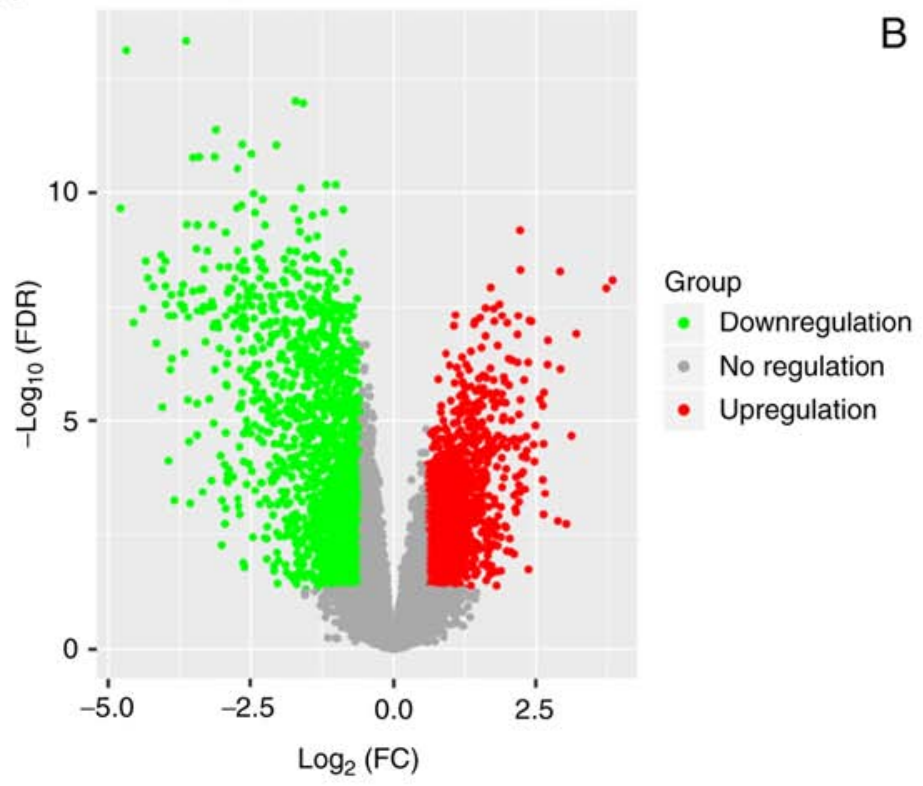

B miRNAs target mRNAs

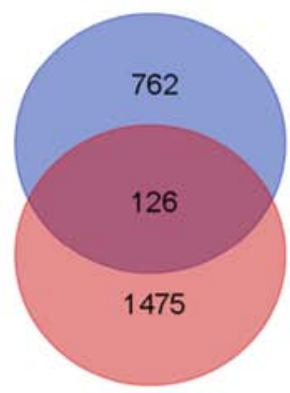

GSE12452 DEGs (upregulated in NPC)

C

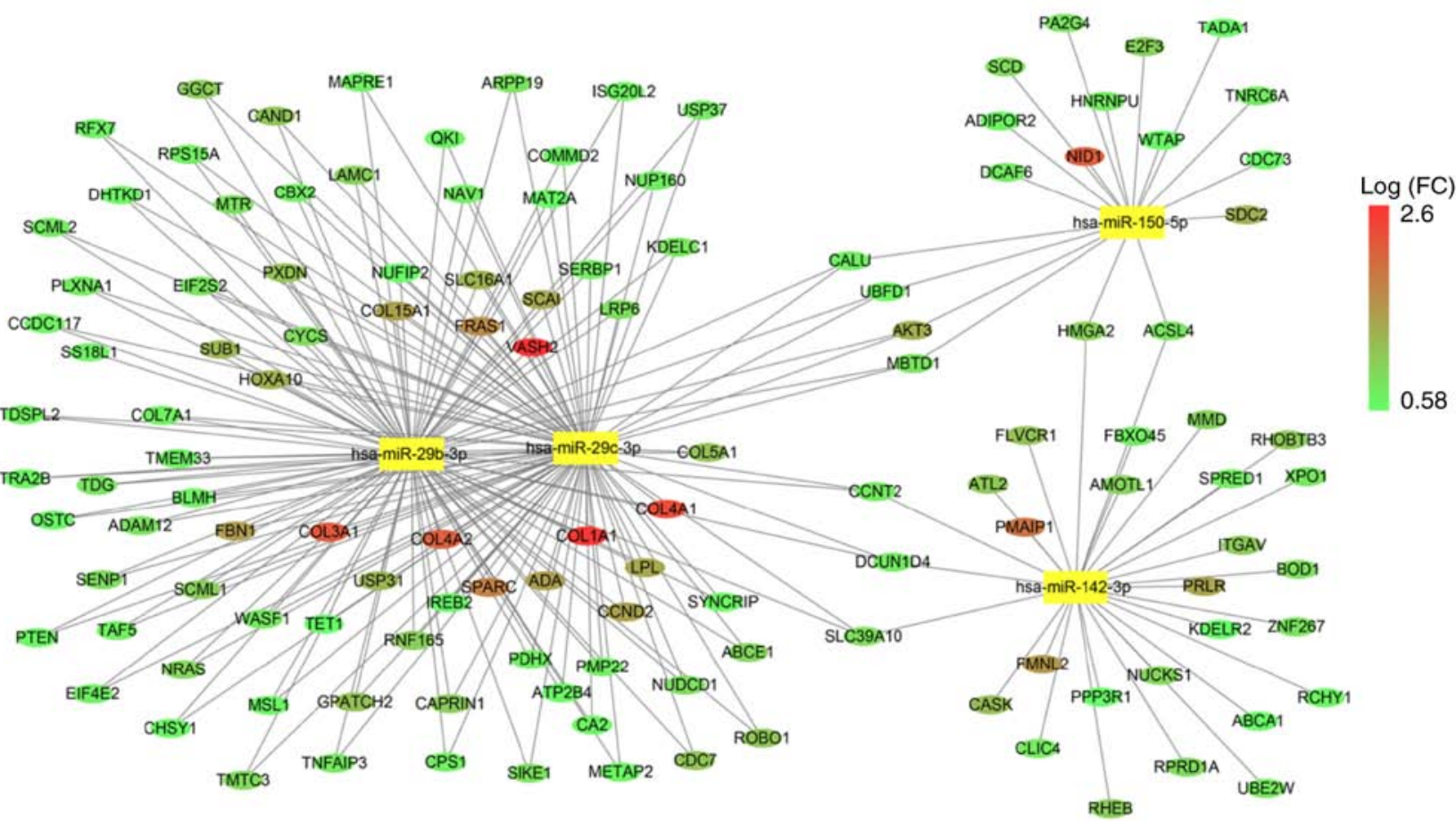

Figure 7. Screening of four miRNA-associated genes. (A) Volcano plot revealing 1,601 upregulated genes (red) and 1,355 downregulated (green) genes in GSE12452 (fold change $>1.5$, adjust P-value (FDR) <0.05). (B) Venn diagram of overlapping mRNAs between 888 upregulated genes in GSE12452 and 1,601 target mRNAs of four hub miRNAs. (C) The miRNA regulatory network constructed with four miRNAs and the screened target mRNAs. The size of mRNA nodes is positively related to the number of their regulatory miRNAs. The colour of mRNA nodes represents their fold change in the GSE12452 dataset. miRNA, microRNA; FDR, false discovery rate.

and extracellular matrix (Table SIX). Biological pathways were mainly enriched in the focal adhesion, PI3K/Akt, p53, mTOR, and ECM-receptor interaction signalling pathways (Table SX).

\section{Discussion}

Prognostic assessment is a crucial part of appropriate treatment choices. In recent years, miRNAs have been reported in the initiation and development of many cancers and are potential biomarkers for diagnosis, prognosis, and personalized treatment $(26,27)$. However, miRNAs as a new biomarker still need to be identified to guide individual treatment for patients. One of the biggest challenges in developing miRNA-based therapeutics is to identify the best miRNA candidates or miRNA targets for each disease type (28). Another major challenge is radioresistance in NPC (29). Hence, to improve the prognosis and clinical treatment of NPC patients, it is urgent to identify crucial prognostic biomarkers. However, by combining miRNAs and mRNAs, the functional properties related to 


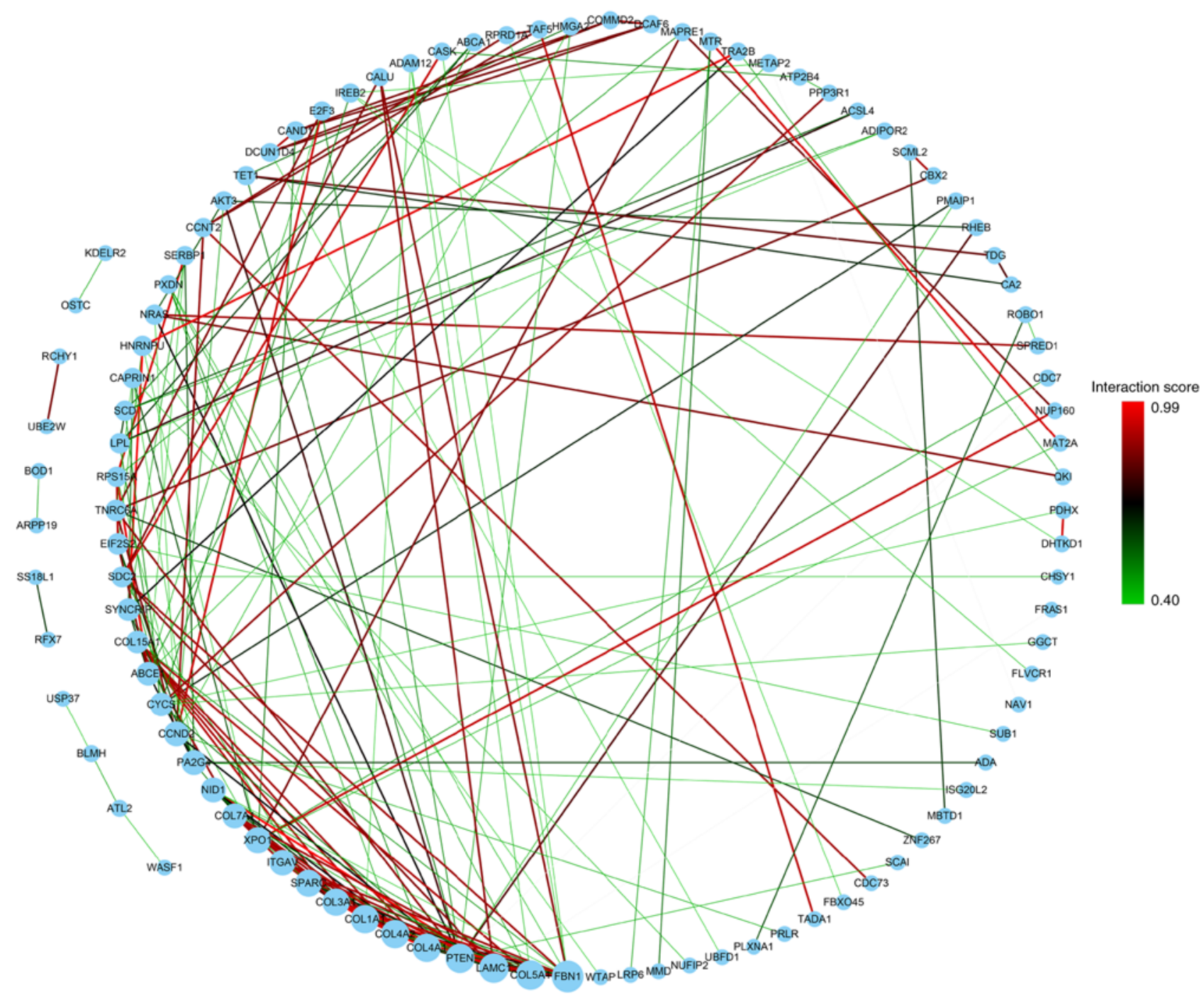

Figure 8. The PPI network of the 127 selected mRNAs. The screened 127 mRNAs were input into the String website for analysis, and just 101 genes have interaction. The size of mRNA nodes is correlated with their interaction number. The colour of the edge reflects the interaction score. PPI, protein-protein interaction.

NPC pathogenesis cannot be determined. Therefore, miRNA expression profiles were analysed in NPC samples relative to normal samples to reveal the potential role of miRNAs in the prognosis of NPC (Figs. 1 and 2).

In the present study, 46 DEMs were identified by analysing the GSE32960 data. In addition, a co-expression network of the associations between clinical traits and the modules was constructed using this dataset, including 312 NPC samples (Figs. 3 and 4). It was revealed that the dark green module was most significantly associated with survival status, such as DMFS, RFS, DFS and OS, by WGCNA and univariate Cox regression analyses. Furthermore, to better understand which of the 46 differentially expressed miRNAs was critical in the development of clinical outcome, univariate Cox regression analysis was performed. In the present study, it was also revealed that four hub miRNAs (hsa-miR-142-3p, hsa-miR-150, hsa-miR-29b, and hsa-miR-29c) were significantly downregulated and positively associated with DFS. Of them, hsa-miR-29c and hsa-miR-142-3p were positively associated with DMFS by Kaplan-Meier survival analysis (Figs. 5 and S3-S5). Subsequently, a four-miRNA signature was constructed to predict the prognosis of NPC patients. The risk score of the four miRNAs revealed a better prediction of survival than did TNM stage, $\mathrm{T}$ stage, $\mathrm{N}$ stage and sex alone with regard to DMFS, OS, RFS, and DFS (Fig. 6). Clinically, NPC is a unique malignancy that is highly invasive and metastatic. It was confirmed that $\sim 50-60 \%$ of patients developed distant metastases during the process of the disease (30). Patients with loco-regionally advanced NPC (stages III and IV) were reported to have a 5 -year survival rate of only $40 \%$ despite treatment with standard RT. In contrast, the great majority of patients died from distant recurrences (31). It has been reported in the literature that mir-29c is downregulated in nasopharyngeal carcinoma and targets a variety of mRNAs, such as extracellular matrix proteins involved in cell migration and metastasis. Increased accumulation of mRNAs encoding proteins may contribute to the invasion and metastasis of NPC (32). Therefore, a decrease in the expression of mir-29c in NPC cells may contribute to its aggressive characteristics. Recently, a study indicated that miR-142-3p, a key suppressive regulator, was epigenetically silenced by DNMT1 and suppressed NPC cell metastasis and EMT by targeting ZEB2 (33). Therefore, miR-142-3p may be 
A

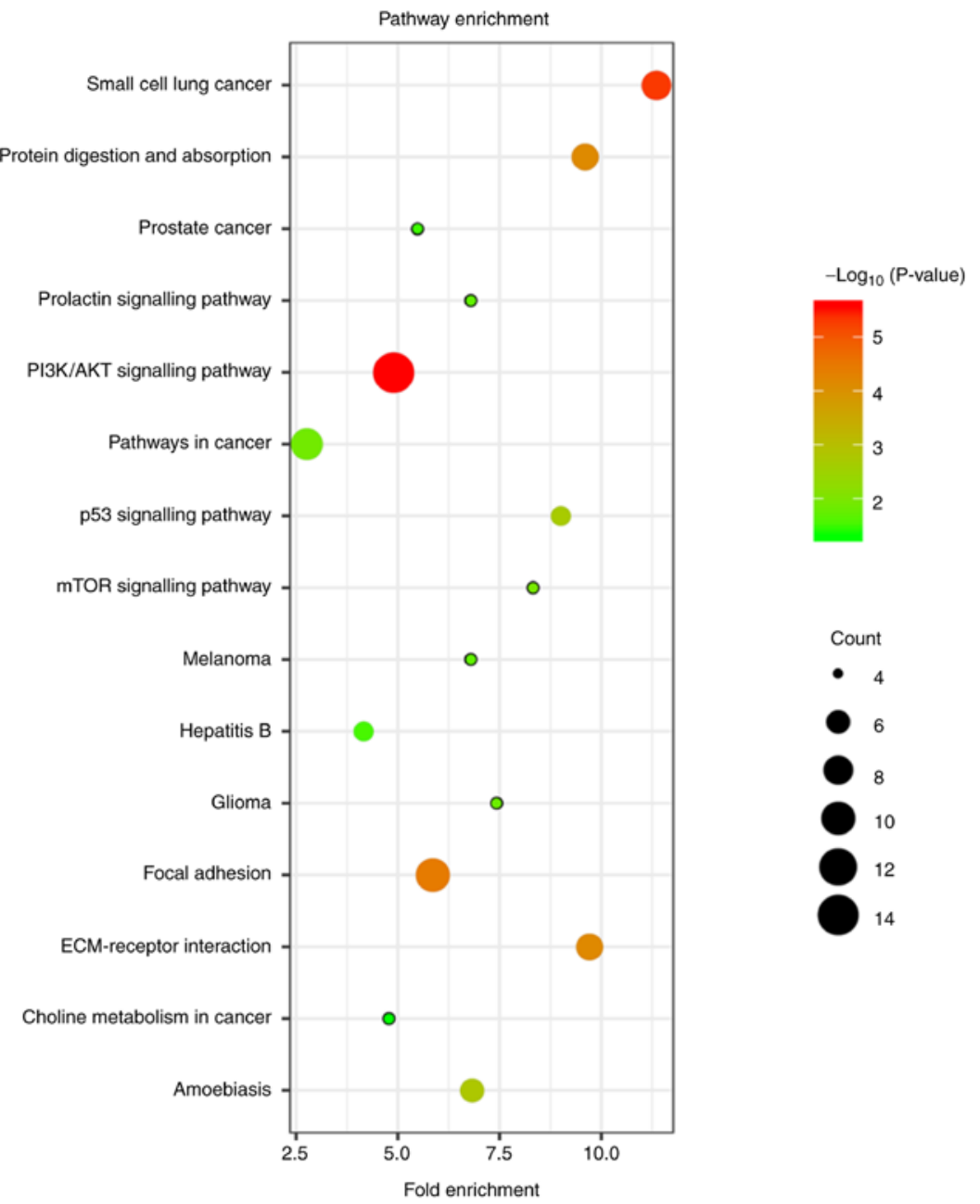

B

Top 20 of GO terms

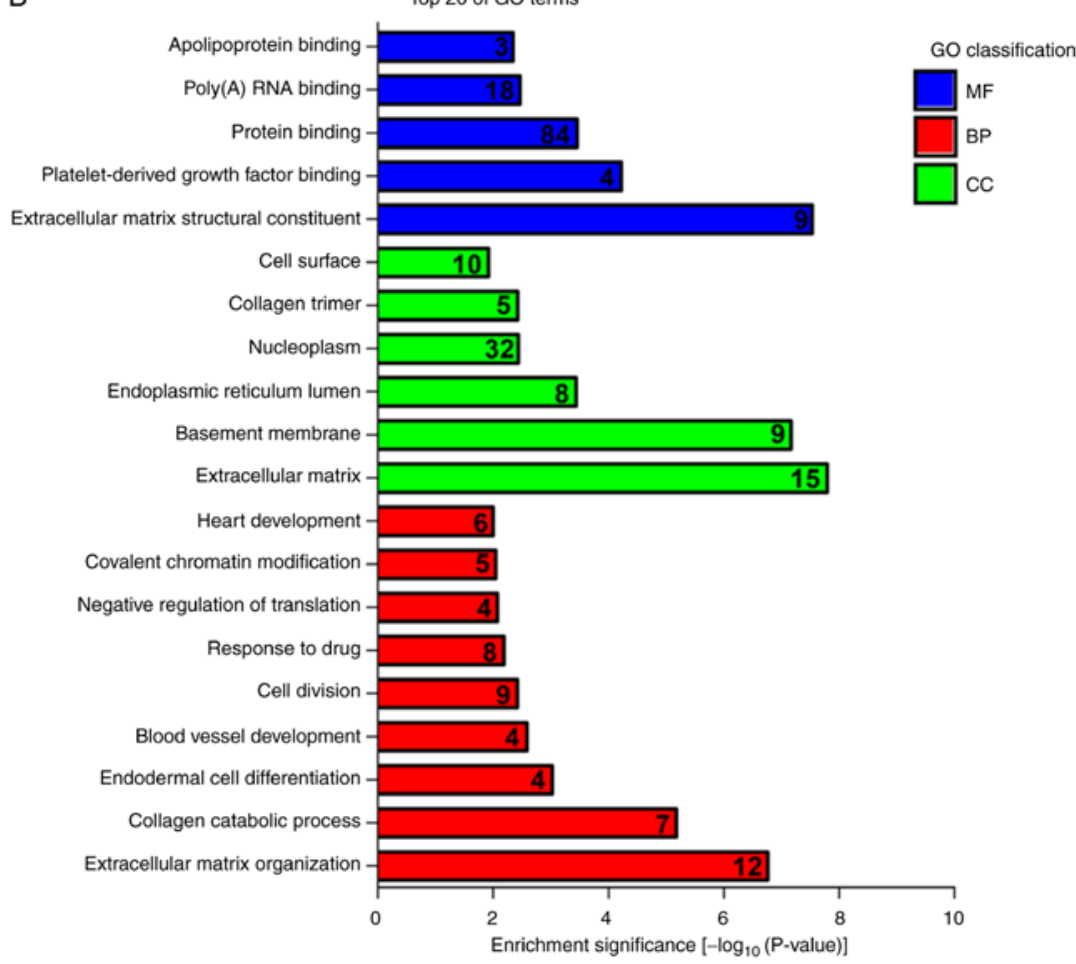

Figure 9. The potential mechanism of four hub miRNAs impacting patient survival. (A) The main pathways of the screened $127 \mathrm{mRNAs}$. The size of the dot reveals the target gene number in the pathway, and the colour of the dot represents the different P-value range. The X-axis reveals the fold enrichment of genes located in the pathway, and the $y$-axis exhibits significantly enriched pathways, $\mathrm{P}<0.05$. The $-\log _{10}(\mathrm{P}$-value) of each term is coloured according to the legend on the right. (B) The top $20 \mathrm{GO}$ terms of the selected 127 genes. Likewise, the x-axis reveals the fold enrichment significance of genes, and the y-axis reveals the GO terms. GO, Gene Ontology; BP, biological process; CC, cellular component; MF, molecular function. 
a potential prognostic marker and therapeutic target to fight against the metastasis of NPC. One study in NPC revealed that miR-150 can modulate the EMT course in NPC/HK-1 cells and lead to cell invasion (34). In addition, miR-29a/b may contribute to the increase in migration and invasion of S18 cells, a type of nasopharyngeal carcinoma cell (35). Furthermore, miRNAs emerging as important modulators in biological pathways can regulate target gene expression and play a key role in tumourigenesis through translational repression or mRNA degradation, indicating that these miRNAs are candidates for clinical applications in the treatment of cancer (36-39). The function and mechanism of the four miRNAs in NPC pathogenesis have not been presented thoroughly. Further investigation into their functions and partners may provide us with more targets and strategies for therapy. Moreover, differentially expressed mRNA data (GSE32960) were integrated with predicted miRNA targets to increase the accuracy of target prediction. Therefore, bioinformatics analysis was applied to explore the potential correlation between miRNAs and mRNA expression profiles. The target genes of downregulated miRNAs in NPC should be upregulated. Accordingly, the 127 shared genes were obtained from 888 targets genes of hub genes and 1,601 upregulated mRNAs as aforementioned. The 127 mRNAs were then identified by superimposing differentially expressed mRNAs and target genes of miRNAs, which were used for subsequent gene functional enrichment analysis (Fig. 7). Increasing evidence has confirmed that miRNAs play an important role in regulating the expression of protein-coding genes (40-42). To understand the potential functional roles of miRNAs, GO and BP analyses were performed. The network revealed that AKT3, PTEN, FBN1, LAMC1, COL5A1, COL1A1, COL4A1, and other genes may play an important role in the interaction (Fig. 8). In particular, the results revealed that their target genes were significantly associated with the focal adhesion, PI3K/Akt, p53, and mTOR signalling pathways (Fig. 9A). In the present study, PTEN, a type of tumour suppressor and a negative regulator of PI3K/Akt-dependent cellular survival, has been implicated in several cancer progressions and was revealed to be involved in p53 signalling (43-46). The aforementioned results indicated a possible role of PTEN in p53 and its related signalling pathways in the dysregulation of miRNAs during NPC pathogenesis (47-49). Of course, miRNAs play a role in tumour suppression in other pathways, and we confirmed that the tumour suppressor miR-216b inhibited the KRAS-related AKT and ERK pathways (50). Therefore, we conclude that the results of our gene functional enrichment analysis are reliably consistent with the present studies.

In conclusion, we successfully identified a four-miRNA signature using an integrated bioinformatics analysis for predicting the prognosis of patients with NPC and then analysed their target genes along with potential biological signalling pathways in the development and progression of NPC. GO and BP analyses enabled the identification of possible associations between miRNAs and protein-coding genes and revealed the potential roles of miRNAs in NPC pathogenesis. Additionally, the greatest advantage in the application of miRNA biology in the clinical management of patients with NPC is its ability to target multiple genes. However, the regulatory roles of the four miRNAs related to p53 signalling or other vital signalling pathways in the genesis and development mechanism of NPC and the detailed regulatory mechanisms still require further study before this four-miRNA signature can be successfully applied clinically. It is our sincere hope that the present study may help promote future individualized treatment of NPC.

\section{Acknowledgements}

We are deeply grateful to all donors who participated in this study.

\section{Funding}

The present study was supported by the National Key Research and Development Program and the National Natural Science Foundations of China (2017YFC1200204, 31670171 and 81728011) and the Innovation Foundations for Graduates of Central South University (2018zzts821).

\section{Availability of data and materials}

The datasets used and/or analyzed during the current study are available from the corresponding authors on request.

\section{Authors' contributions}

SZ conceived, designed, and performed the statistical analysis and wrote the paper. WY and SL participated in analyzing the data, performing the statistical analysis and drafting the manuscript. LY, XZ and PC helped to analyze the data. YX, LL, WD and SX provisioned suggestions in figure preparation and critically revised the manuscript. JL conceived and supervised the study. All authors have read and approved the final version of the manuscript and agree to be accountable for all aspects of the work in ensuring that questions related to the accuracy or integrity of any part of the work are appropriately investigated and resolved.

\section{Ethics approval and consent to participate}

Not applicable.

\section{Patient consent for publication}

Not applicable.

\section{Competing interests}

The authors declare that they have no competing interests.

\section{References}

1. Torre LA, Bray F, Siegel RL, Ferlay J, Lortet-Tieulent J and Jemal A: Global cancer statistics, 2012. CA Cancer J Clin 65: 87-108, 2015.

2. Pan JJ, Ng WT, Zong JF, Lee SW, Choi HC, Chan LL, Lin SJ, Guo QJ, Sze HC, Chen YB, et al: Prognostic nomogram for refining the prognostication of the proposed 8th edition of the AJCC/UICC staging system for nasopharyngeal cancer in the era of intensity-modulated radiotherapy. Cancer 122: 3307-3315, 2016. 
3. Hui EP, Leung SF, Au JS, Zee B, Tung S, Chua D, Sze WM, Law CK, Leung TW and Chan AT: Lung metastasis alone in nasopharyngeal carcinoma: A relatively favorable prognostic group. A study by the Hong Kong nasopharyngeal carcinoma study group. Cancer 101: 300-306, 2004.

4. Ng WT, Yuen KT, Au KH, Chan OS and Lee AW: Staging of nasopharyngeal carcinoma-the past, the present and the future. Oral Oncol 50: 549-554, 2014.

5. Lin JC, Chen KY, Wang WY, Jan JS, Liang WM, Tsai CS and Wei YH: Detection of Epstein-Barr virus DNA in the peripheral-blood cells of patients with nasopharyngeal carcinoma: Relationship to distant metastasis and survival. J Clin Oncol 19: 2607-2615, 2001.

6. Zhou GQ, Tang LL, Mao YP, Chen L, Li WF, Sun Y, Liu LZ, $\mathrm{Li} \mathrm{L}$, Lin $\mathrm{AH}$ and Ma J: Baseline serum lactate dehydrogenase levels for patients treated with intensity-modulated radiotherapy for nasopharyngeal carcinoma: A predictor of poor prognosis and subsequent liver metastasis. Int J Radiat Oncol Biol Phys 82 e359-e365, 2012

7. Lv X, Xiang YQ, Cao SM, Qian CN, Li NW, Guo L, Mai HQ, Chen QY, Huang PY, Luo D, et al: Prospective validation of the prognostic value of elevated serum vascular endothelial growth factor in patients with nasopharyngeal carcinoma: More distant metastases and shorter overall survival after treatment. Head Neck 33: 780-785, 2011

8. Tang XR, Li YQ, Liang SB, Jiang W, Liu F, Ge WX, Tang LL, Mao YP, He QM, Yang XJ, et al: Development and validation of a gene expression-based signature to predict distant metastasis in locoregionally advanced nasopharyngeal carcinoma: A retrospective, multicentre, cohort study. Lancet Oncol 19: 382-393, 2018.

9. Goretti E, Wagner DR and Devaux Y: miRNAs as biomarkers of myocardial infarction: A step forward towards personalized medicine? Trends Mol Med 20: 716-725, 2014

10. Deng Z, Wang Y, Fang X, Yan F, Pan H, Gu L, Xie C, Li Y, $\mathrm{Hu}$ Y, Cao Y and Tang Z: Research on miRNA-195 and target gene CDK6 in oral verrucous carcinoma. Cancer Gene Ther 24: 282-288, 2017.

11. He B, Li W, Wu Y, Wei F, Gong Z, Bo H, Wang Y, Li X, Xiang B, Guo C, et al: Epstein-Barr virus-encoded miR-BART6-3p inhibits cancer cell metastasis and invasion by targeting long non-coding RNA LOC553103. Cell Death Dis 7: e2353, 2016.

12. Watanabe A, Tagawa $H$, Yamashita J Teshima $K$, Nara M, Iwamoto K, Kume M, Kameoka Y, Takahashi N, Nakagawa T, et al: The role of microRNA-150 as a tumor suppressor in malignant lymphoma. Leukemia 25: 1324-1334, 2011.

13. Saito Y, Suzuki H, Imaeda H, Matsuzaki J, Hirata K, Tsugawa $H$, Hibino S, Kanai Y, Saito H and Hibi T: The tumor suppressor microRNA-29c is downregulated and restored by celecoxib in human gastric cancer cells. Int J Cancer 132: 1751-1760, 2013.

14. Fang JH, Zhou HC, Zeng C, Yang J, Liu Y, Huang X, Zhang JP, Guan XY and Zhuang SM: MicroRNA-29b suppresses tumor angiogenesis, invasion, and metastasis by regulating matrix metalloproteinase 2 expression. Hepatology 54: 1729-1740, 2011.

15. Lee KT, Tan JK, Lam AK and Gan SY: MicroRNAs serving as potential biomarkers and therapeutic targets in nasopharyngeal carcinoma: A critical review. Crit Rev Oncol Hematol 103: 1-9, 2016.

16. Liu N, Chen NY, Cui RX, Li WF, Li Y, Wei RR, Zhang MY, Sun Y, Huang BJ, Chen M, et al: Prognostic value of a microRNA signature in nasopharyngeal carcinoma: A microRNA expression analysis. Lancet Oncol 13: 633-641, 2012.

17. Sengupta S, den Boon JA, Chen IH, Newton MA, Dahl DB, Chen M, Cheng YJ, Westra WH, Chen CJ, Hildesheim A, et al: Genome-wide expression profiling reveals EBV-associated inhibition of MHC class I expression in nasopharyngeal carcinoma Cancer Res 66: 7999-8006, 2006

18. Horvath S and Dong J: Geometric interpretation of gene coexpression network analysis. PLoS Comput Biol 4: e1000117, 2008

19. Langfelder $P$ and Horvath S: WGCNA: An R package for weighted correlation network analysis. BMC Bioinformatics 9: 559, 2008.

20. Yang JH, Li JH, Shao P, Zhou H, Chen YQ and Qu LH: starBase: A database for exploring microRNA-mRNA interaction maps from Argonaute CLIP-Seq and Degradome-Seq data. Nucleic Acids Res 39 (Database Issue): D202-D209, 2011.

21. Dennis G, Jr, Sherman BT, Hosack DA, Yang J, Gao W, Lane HC and Lempicki RA: DAVID: Database for annotation, visualization, and integrated discovery. Genome Biol 4: P3, 2003.
22. Szklarczyk D, Franceschini A, Wyder S, Forslund K, Heller D, Huerta-Cepas J, Simonovic M, Roth A, Santos A, Tsafou KP, et al: STRING v10: Protein-protein interaction networks, integrated over the tree of life. Nucleic Acids Res 43 (Database Issue): D447-D452, 2015.

23. Shannon P, Markiel A, Ozier O, Baliga NS, Wang JT, Ramage D, Amin N, Schwikowski B and Ideker T: Cytoscape: A software environment for integrated models of biomolecular interaction networks. Genome Res 13: 2498-2504, 2003.

24. Yu SL, Chen HY, Chang GC, Chen CY, Chen HW, Singh S, Cheng CL, Yu CJ, Lee YC, Chen HS, et al: MicroRNA signature predicts survival and relapse in lung cancer. Cancer Cell 13: 48-57, 2008.

25. Lossos IS, Czerwinski DK, Alizadeh AA, Wechser MA, Tibshirani R, Botstein D and Levy R: Prediction of survival in diffuse large-B-cell lymphoma based on the expression of six genes. N Engl J Med 350: 1828-1837, 2004.

26. Ueda T, Volinia S, Okumura H, Shimizu M, Taccioli C, Rossi S, Alder H, Liu CG, Oue N, Yasui W, et al: Relation between microRNA expression and progression and prognosis of gastric cancer: A microRNA expression analysis. Lancet Oncol 11: $136-146,2010$.

27. Ji J, Shi J, Budhu A, Yu Z, Forgues M, Roessler S, Ambs S, Chen Y, Meltzer PS, Croce CM, et al: MicroRNA expression, survival, and response to interferon in liver cancer. N Engl J Med 361: 1437-1447, 2009.

28. Li Z and Rana TM: Therapeutic targeting of microRNAs: Current status and future challenges. Nat Rev Drug Discov 13: 622-638, 2014

29. Qu JQ, Yi HM, Ye X, Zhu JF, Yi H, Li LN, Xiao T, Yuan L, Li JY, Wang YY, et al: miRNA-203 reduces nasopharyngeal carcinoma radioresistance by targeting IL8/AKT signaling. Mol Cancer Ther 14: 2653-2664, 2015.

30. Cvitkovic E, Bachouchi M, Boussen H, Busson P, Rousselet G, Mahjoubi R, Flores P, Tursz T, Armand JP and Azli N: Leukemoid reaction, bone marrow invasion, fever of unknown origin, and metastatic pattern in the natural history of advanced undifferentiated carcinoma of nasopharyngeal type: A review of 255 consecutive cases. J Clin Oncol 11: 2434-2442, 1993.

31. Teo PM, Kwan WH, Lee WY, Leung SF and Johnson PJ: Prognosticators determining survival subsequent to distant metastasis from nasopharyngeal carcinoma. Cancer 77: 2423-2431, 1996

32. Sengupta S, den Boon JA, Chen IH, Newton MA, Stanhope SA, Cheng YJ, Chen CJ, Hildesheim A, Sugden B and Ahlquist P: MicroRNA $29 \mathrm{c}$ is down-regulated in nasopharyngeal carcinomas, up-regulating mRNAs encoding extracellular matrix proteins. Proc Natl Acad Sci USA 105: 5874-5878, 2008.

33. Li Y, He Q, Wen X, Hong X, Yang X, Tang X, Zhang P, Lei Y, Sun Y, Zhang J, et al: EZH2-DNMT1-mediated epigenetic silencing of miR-142-3p promotes metastasis through targeting ZEB2 in nasopharyngeal carcinoma. Cell Death Differ 26: 1089-1106, 2019

34. Yue PY, Ha WY, Lau CC, Cheung FM, Lee AW, Ng WT, Ngan RK, Yau CC, Kwong DL, Lung HL, et al: MicroRNA profiling study reveals miR-150 in association with metastasis in nasopharyngeal carcinoma. Sci Rep 7: 12012, 2017.

35. Qiu F, Sun R, Deng N, Guo T, Cao Y, Yu Y, Wang X, Zou B, Zhang S, Jing T, et al: miR-29a/b enhances cell migration and invasion in nasopharyngeal carcinoma progression by regulating SPARC and COL3A1 gene expression. PLoS One 10: e0120969, 2015.

36. Zheng Z, Qu JQ, Yi HM, Ye X, Huang W, Xiao T, Li JY, Wang YY, Feng J, Zhu JF, et al: miR-125b regulates proliferation and apoptosis of nasopharyngeal carcinoma by targeting A20/NF- $\mathrm{KB}$ signaling pathway. Cell Death Dis 8: e2855, 2017.

37. Tan G, Tang X and Tang F: The role of microRNAs in nasopharyngeal carcinoma. Tumour Biol 36: 69-79, 2015.

38. Cho WC: MicroRNAs in cancer-from research to therapy. Biochim Biophys Acta 1805: 209-217, 2010.

39. Zuo LL, Zhang J, Liu LZ, Zhou Q, Du SJ, Xin SY, Ning ZP, Yang J, Yu HB, Yue WX, et al: Cadherin 6 is activated by Epstein-Barr virus LMP1 to mediate EMT and metastasis as an interplay node of multiple pathways in nasopharyngeal carcinoma. Oncogenesis 6: 402, 2017.

40. Lin CW, Li XR, Zhang Y, Hu G, Guo YH, Zhou JY, Du J, Lv L, Gao K, Zhang Y and Deng H: TAp63 suppress metastasis via miR-133b in colon cancer cells. Br J Cancer 110: 2310-2320, 2014. 
41. Yu H, Lu J, Zuo L, Yan Q, Yu Z, Li X, Huang J, Zhao L, Tang H, Luo Z, et al: Epstein-Barr virus downregulates microRNA 203 through the oncoprotein latent membrane protein 1: A contribution to increased tumor incidence in epithelial cells. J Virol 86: 3088-3099, 2012.

42. Peng G, Liao Y and Shen C: miRNA-429 inhibits astrocytoma proliferation and invasion by targeting BMI1. Pathol Oncol Res 23: 369-376, 2017.

43. Mayo LD and Donner DB: The PTEN, Mdm2, p53 tumor suppressor-oncoprotein network. Trends Biochem Sci 27: 462-467, 2002.

44. Stambolic V, MacPherson D, Sas D, Lin Y, Snow B, Jang Y, Benchimol S and Mak TW: Regulation of PTEN transcription by p53. Mol Cell 8: 317-325, 2001.

45. Freeman DJ, Li AG, Wei G, Li HH, Kertesz N, Lesche R, Whale AD, Martinez-Diaz H, Rozengurt N, Cardiff RD, et al: PTEN tumor suppressor regulates p53 protein levels and activity through phosphatase-dependent and -independent mechanisms. Cancer Cell 3: 117-130, 2003.

46. Jung SH, Hwang HJ, Kang D, Park HA, Lee HC, Jeong D, Lee K, Park HJ, Ko YG and Lee JS: mTOR kinase leads to PTEN-loss-induced cellular senescence by phosphorylating p53. Oncogene 38: 1639-1650, 2019.
47. Andreozzi M, Quagliata L, Gsponer JR, Ruiz C, Vuaroqueaux V, Eppenberger-Castori S, Tornillo L and Terracciano LM: VEGFA gene locus analysis across 80 human tumour types reveals gene amplification in several neoplastic entities. Angiogenesis 17: 519-527, 2014.

48. Chen HX, Xu XX, Tan BZ, Zhang Z and Zhou XD: MicroRNA-29b inhibits angiogenesis by targeting VEGFA through the MAPK/ERK and PI3K/Akt signaling pathways in endometrial carcinoma. Cell Physiol Biochem 41: 933-946, 2017.

49. Cheng JZ, Chen JJ, Xue K, Wang ZG and Yu D: Clinicopathologic and prognostic significance of VEGF, JAK2 and STAT3 in patients with nasopharyngeal carcinoma. Cancer Cell Int 18: 110, 2018.

50. Deng M, Tang H, Zhou Y, Zhou M, Xiong W, Zheng Y, Ye Q, Zeng X, Liao Q, Guo X, et al: miR-216b suppresses tumor growth and invasion by targeting KRAS in nasopharyngeal carcinoma. J Cell Sci 124: 2997-3005, 2011.

This work is licensed under a Creative Commons Attribution-NonCommercial-NoDerivatives 4.0 International (CC BY-NC-ND 4.0) License. 